\title{
Thermodynamic and Energetic Limits on Continental Silicate Weathering Strongly Impact the Climate and Habitability of Wet, Rocky Worlds
}

\author{
R. J. Graham (10) and Ray Pierrehumbert \\ University of Oxford, UK; robert.graham@ physics.ox.ac.uk \\ Received 2020 February 25; revised 2020 May 1; accepted 2020 May 13; published 2020 June 18
}

\begin{abstract}
The "liquid water habitable zone" (HZ) concept is predicated on the ability of the silicate weathering feedback to stabilize climate across a wide range of instellations. However, representations of silicate weathering used in current estimates of the effective outer edge of the $\mathrm{HZ}$ accounts for neither the thermodynamic limit on the concentration of weathering products in runoff set by clay precipitation nor the energetic limit on precipitation set by planetary instellation. We find that when the thermodynamic limit is included in an idealized coupled climate/ weathering model, the steady-state planetary climate loses sensitivity to silicate dissolution kinetics, becoming sensitive to temperature primarily through the effect of temperature on runoff and to $\mathrm{pCO}_{2}$ through an effect on solute concentration mediated by $\mathrm{pH}$. This increases sensitivity to land fraction, $\mathrm{CO}_{2}$ outgassing, and geological factors such as soil age and lithology, all of which are found to have a profound effect on the position of the effective outer edge of the HZ. The interplay between runoff sensitivity and the energetic limit on precipitation leads to novel warm states in the outer reaches of the HZ, owing to the decoupling of temperature and precipitation. We discuss strategies for detecting the signature of the silicate weathering feedback through exoplanet observations in light of insights derived from the revised picture of weathering.
\end{abstract}

Unified Astronomy Thesaurus concepts: Habitable planets (695); Habitable zone (696); Exoplanet atmospheres (487); Exoplanet evolution (491)

\section{Introduction}

The classical "liquid water habitable zone" (HZ) is the orbital region around stars where rocky planets with $\mathrm{N}_{2}-\mathrm{H}_{2} \mathrm{O}-\mathrm{CO}_{2}$ atmospheres can potentially support stable liquid water on their surfaces (Huang 1959; Hart 1979; Kasting et al. 1993; Kopparapu et al. 2013; Ramirez 2018). The inner edge of the $\mathrm{HZ}$ is the instellation at which a planet's oceans are likely to undergo rapid escape to space, either due to enhanced loss of water from a warm, wet stratosphere (the "moist greenhouse state") or due to full ocean evaporation and subsequent escape (the "runaway greenhouse state"; e.g., Kasting 1988; Kasting et al. 1993; Kopparapu et al. 2013). The outer edge of the $\mathrm{HZ}$ is the instellation at which a planet cannot maintain a temperature above the freezing point of water anywhere on its surface with an atmosphere of $\mathrm{N}_{2}, \mathrm{H}_{2} \mathrm{O}$, and $\mathrm{CO}_{2}$ alone (e.g., Kadoya \& Tajika 2019). Depending on conditions like the spectrum of the host star, the outer edge may be defined by the "maximum greenhouse limit," where an increase in the $\mathrm{CO}_{2}$ partial pressure on a hypothetical planet would increase the Rayleigh scattering albedo enough to outweigh the greenhouse effect and cause the planet to freeze, or it may be defined by the " $\mathrm{CO}_{2}$ condensation limit," where $\mathrm{CO}_{2}$ can no longer accumulate to higher levels in the atmosphere because it is forced to condense or deposit at the planet's surface (e.g., Kasting et al. 1993; Kopparapu 2013).

The HZ concept as currently understood relies on the existence of a negative feedback mechanism on ocean-bearing rocky planets to control the concentration of atmospheric $\mathrm{CO}_{2}$ and maintain surface liquid water at a variety of instellations. Without some kind of long-term negative feedback on climate, the question of whether a given planet's atmospheric $\mathrm{CO}_{2}$ level is consistent with the presence of surface liquid $\mathrm{H}_{2} \mathrm{O}$ at that planet's particular instellation is largely a matter of luck, and the lifetime of habitability for those planets lucky enough to start off temperate will be relatively short, due to the gradual brightening of stars. Walker et al. (1981; WHAK) suggested that temperature-, runoff-, and $\mathrm{pCO}_{2}$-dependent weathering of silicate minerals (a process that produces cations that react with oceanic carbonate ions to form carbonate minerals that are sequestered in Earth's crust and subsequently recycled through the mantle) has served the role of stabilizing feedback on Earth, drawing down $\mathrm{CO}_{2}$ as the Sun has brightened from $\sim 70 \%$ of its current luminosity over the past 4 billion years $(\mathrm{Ga}$; Sagan \& Mullen 1972; Bahcall et al. 2001). Kasting et al. (1988) hypothesized that this silicate weathering feedback also operates on rocky ocean- and land-bearing exoplanets, and this idea underpins much subsequent work regarding the $\mathrm{HZ}$ (though see, e.g., Pierrehumbert \& Gaidos 2011, Kite \& Ford 2018, and Ramirez \& Levi 2018 for nonstandard habitability scenarios that do not involve the silicate weathering feedback). Recently, studies modeling planetary weathering behavior have suggested that enhanced weathering due to high $\mathrm{pCO}_{2}$ in the outer reaches of the $\mathrm{HZ}$ may act to draw $\mathrm{CO}_{2}$ down to levels that force a planet into the snowball state at instellations greater than the "maximum greenhouse limit" or " $\mathrm{CO}_{2}$ condensation limit," effectively decreasing the width of the $\mathrm{HZ}$, but the magnitude (and even the existence) of this effect is strongly dependent on the details of the silicate weathering feedback (Menou 2015; Abbot 2016; Haqq-Misra et al. 2016; Kadoya \& Tajika 2019). Accurately representing silicate weathering in models is therefore necessary for predicting the extent of the $\mathrm{HZ}$ and the behavior of planets within it. These issues will become increasingly crucial as observation and analysis of temperate rocky planets and their atmospheres by future telescopes become commonplace. Here, we focus on continental weathering, though a full evaluation of habitability would, among other things, require a treatment of 
seafloor weathering as well (see Section 5.1.6 for discussion of this issue).

In this study, we use a simple zero-dimensional climate model and the weathering framework developed in Maher \& Chamberlain (2014; MAC) to reexamine planetary weathering behavior and the outer edge of the HZ. The MAC model of weathering is the first to include a thermodynamic limit on cation concentration in runoff due to equilibration between dissolving silicates and precipitating clays. We also include an energetic limit on precipitation and runoff that accounts for the fact that evaporation (and therefore precipitation) is ultimately driven by instellation, which implies the existence of a maximum precipitation rate for a planet, defined as the rate at which all incoming stellar radiation must be used for the latent heat of evaporation (Pierrehumbert 2002; O'Gorman \& Schneider 2008). We find that including these limits in global weathering models strongly impacts the climate and weathering behavior of wet, rocky exoplanets in the HZ. In particular, including the thermodynamic limit on solute concentration increases the importance of hydrology and surface properties like land fraction and soil age for global weathering fluxes, alternately expanding or contracting the effective width of the HZ depending on choice of parameters. With the energetic limit on precipitation, global precipitation ceases to be a function of temperature under some circumstances, leading to fundamental changes in the functioning of the silicate weathering feedback.

In the remainder of this paper, we will more thoroughly introduce the silicate weathering feedback, along with its thermodynamic and energetic limits, which have not been included in previous studies of exoplanet habitability (Section 2); describe the simple models we used to examine the impact of these limits on the behavior of planets within the HZ (Section 3); present the results of our calculations (Section 4); and discuss implications of these results (Section 5).

\section{The Continental Silicate Weathering Feedback}

The continental silicate weathering feedback is a mechanism proposed by WHAK to explain how Earth has maintained a relatively stable, temperate climate over geologic time despite volcanic outgassing of $\mathrm{CO}_{2}$ and the long-term brightening of the Sun. In Earth's carbonate-silicate cycle (e.g., Siever 1968; Walker et al. 1981), volcanic $\mathrm{CO}_{2}$ is released into the atmosphere/ocean system by the metamorphism and melting of carbonate minerals in the planet's interior. $\mathrm{CO}_{2}$ acts as an acid in aqueous silicate weathering reactions that speed up the release of $\mathrm{Ca}^{2+}$ and $\mathrm{Mg}^{2+}$ cations, which are then carried by rivers to the oceans, where they react with carbonate ions and precipitate as carbonate minerals. These minerals sink to the ocean floor, where they are subducted into Earth, completing the cycle. This complex process can be represented schematically as

$$
\mathrm{CaSiO}_{3}+\mathrm{CO}_{2} \leftrightarrow \mathrm{CaCO}_{3}+\mathrm{SiO}_{2},
$$

where the rightward direction represents a silicate mineral reacting with (and consuming) a $\mathrm{CO}_{2}$ molecule to form a carbonate mineral and silica $\left(\mathrm{SiO}_{2}\right)$, and the leftward direction represents an overall reaction where a carbonate mineral is metamorphosed with silica in Earth's mantle to form a silicate and $\mathrm{CO}_{2}$. In actuality, a variety of silicate minerals can take the place of $\mathrm{CaSiO}_{3}$ in the above equation, and secondary minerals, e.g., clays, precipitate in regions being weathered and play vital roles in controlling the rates of the weathering reactions (Alekseyev et al. 1997; Maher et al. 2009; Maher \& Chamberlain 2014), with important implications to be discussed in Section 2.2.

\subsection{The WHAK Model}

To understand where negative feedback enters into this process in the WHAK model, we can isolate the step where the silicate mineral is dissolved:

$$
\begin{aligned}
& \mathrm{CaSiO}_{3}(\mathrm{~s})+2 \mathrm{CO}_{2}(\mathrm{~g})+\mathrm{H}_{2} \mathrm{O}(\mathrm{l}) \rightarrow \\
& \mathrm{Ca}^{2+}(\mathrm{aq})+2 \mathrm{HCO}_{3}^{-}(\mathrm{aq})+\mathrm{SiO}_{2}(\mathrm{aq}) .
\end{aligned}
$$

Laboratory studies demonstrate that the kinetics of silicate dissolution depend directly on temperature and $\mathrm{pH}$ for a variety of silicate minerals (e.g., Schott \& Berner 1985; Brady 1991; Knauss et al. 1993; Oxburgh et al. 1994; Welch \& Ullman 1996; Chen \& Brantley 1998; Weissbart \& Rimstidt 2000; Oelkers \& Schott 2001; Palandri \& Kharaka 2004; Carroll \& Knauss 2005; Golubev et al. 2005; Bandstra \& Brantley 2008; Brantley et al. 2008). Atmospheric $\mathrm{pCO}_{2}$ influences temperature through its greenhouse effect and $\mathrm{pH}$ through its action as a weak acid in aqueous solution and its fertilizing effect on plants, which in turn produce organic acids in soils (Brady 1991; Brady \& Carroll 1994). In the remainder of this study, we ignore the impacts of life on weathering and focus on the ability of abiotic planets to achieve stable, temperate climates, as the impact of organisms on weathering may vary immensely from planet to planet, depending on the specifics of metabolism and biogeochemical pathways. Increasing the $\mathrm{CO}_{2}$ partial pressure of the atmosphere warms the planet and reduces the $\mathrm{pH}$ of rainwater, both of which should accelerate silicate dissolution under conditions where the kinetics of dissolution is relevant. This increases the delivery of $\mathrm{Ca}^{2+}\left(\right.$ or $\mathrm{Mg}^{2+}$ ) cations to the ocean, which accelerates the consumption of $\mathrm{CO}_{2}$ and decreases $\mathrm{pCO}_{2}$ until the rate of $\mathrm{CO}_{2}$ consumption by silicate weathering is again equal to the rate of $\mathrm{CO}_{2}$ production by outgassing. The opposite takes place in the case of a $\mathrm{CO}_{2}$ reduction, so silicate weathering can act as a negative feedback to changes in the climate.

The temperature dependence of silicate dissolution rates is usually represented as a simplified Arrhenius law:

$$
r \propto \exp \left(\frac{T-T_{\text {ref }}}{T_{e}}\right)
$$

where $r$ is the silicate dissolution rate, $T$ is the temperature at which dissolution is taking place, and $T_{\text {ref }}$ is a reference temperature. $T_{e}=T_{\mathrm{ref}}^{2} R / E_{\mathrm{act}}$ is the temperature change required to increase or decrease the dissolution rate by a factor of $e$, where $R$ is the ideal gas constant and $E_{\text {act }}$ is the activation energy of the dissolution reaction. Under kinetically limited conditions, higher temperatures accelerate the dissolution of silicate minerals.

The $\mathrm{pH}$ sensitivity of silicate dissolution is more complicated, as the magnitude and sign of the dependence often depend on the $\mathrm{pH}$ itself (e.g., Brantley et al. 2008). Under alkaline conditions, reduced $\mathrm{pH}$ often inhibits dissolution rates; near neutral conditions, the effect of $\mathrm{pH}$ is weak or nonexistent; 
Table 1

List of Parameters Used in This Study

\begin{tabular}{|c|c|c|c|}
\hline Parameter & Units & Definition & Default Value \\
\hline$\gamma$ & $\cdots$ & Land fraction & 0.3 \\
\hline$a_{g}$ & $\cdots$ & Surface albedo & 0.2 \\
\hline$a$ & $\cdots$ & Planetary albedo & 0.3 \\
\hline$R_{\text {planet }}$ & $\begin{array}{l}\text { meters } \\
(\mathrm{m})\end{array}$ & Planetary radius & $6.37 \times 10^{6}$ \\
\hline$\gamma_{\text {ref }}$ & $\cdots$ & $\begin{array}{l}\text { Modern land } \\
\text { fraction }\end{array}$ & 0.3 \\
\hline$T_{\text {ref }}$ & $\begin{array}{l}\text { Kelvin } \\
(\mathrm{K})\end{array}$ & $\begin{array}{l}\text { Modern global- } \\
\text { avg. temperature }\end{array}$ & 288 \\
\hline $\mathrm{pCO}_{2, \text { ref }}$ & bar & $\begin{array}{c}\text { Preindustrial } \\
\mathrm{CO}_{2} \text { partial pressure }\end{array}$ & $280 \times 10^{-6}$ \\
\hline$p_{\text {ref }}$ & $\mathrm{m} \mathrm{yr}^{-1}$ & $\begin{array}{l}\text { Modern global- } \\
\text { avg. precipitation }\end{array}$ & $\begin{array}{c}0.99 \\
\text { (Xie \& Arkin 1997) }\end{array}$ \\
\hline$q_{\mathrm{ref}}$ & $\mathrm{m} \mathrm{yr}^{-1}$ & $\begin{array}{l}\text { Modern global- } \\
\text { avg. runoff }\end{array}$ & $\begin{array}{c}0.20 \\
\text { (Oki et al. 2001) }\end{array}$ \\
\hline$\Gamma$ & $\cdots$ & $\begin{array}{l}\text { Fraction of precip. } \\
\text { that becomes runoff }\end{array}$ & $q_{\mathrm{ref}} / p_{\mathrm{ref}}=0.2$ \\
\hline$\epsilon$ & $1 / \mathrm{K}$ & $\begin{array}{l}\text { Fractional change in } \\
\text { precip. per } \mathrm{K} \\
\text { change in temp. }\end{array}$ & 0.03 \\
\hline$V_{\text {ref }}$ & $\mathrm{mol} \mathrm{yr}^{-1}$ & $\begin{array}{l}\text { Modern global } \\
\mathrm{CO}_{2} \text { outgassing }\end{array}$ & $\begin{array}{c}7.5 \times 10^{12} \\
\text { (Gerlach } 2011 ; \text { Haqq-Misra } \\
\text { et al. 2016) }\end{array}$ \\
\hline$v$ & $\mathrm{~mol} \mathrm{~m}_{\mathrm{yr}^{-1}}^{-2}$ & $\begin{array}{l}\text { Modern } \mathrm{CO}_{2} \\
\text { outgassing } \\
\text { per } \mathrm{m}^{2} \text { plane- } \\
\text { tary area }\end{array}$ & $V_{\text {ref }} / 4 \pi R_{\text {planet }}^{2}=0.0147$ \\
\hline$w_{w, \text { ref }}$ & $\underset{\mathrm{yr}^{-1}}{\mathrm{~mol} \mathrm{~m}^{-2}}$ & $\begin{array}{l}\text { Modern weathering } \\
\text { per } \mathrm{m}^{2} \text { planetary } \\
\text { area }\end{array}$ & $V_{\text {ref }} / 4 \pi R_{\text {planet }}^{2}=0.0147$ \\
\hline$\Lambda$ & variable & $\begin{array}{l}\text { Thermodynamic } \\
\text { coefficient } \\
\text { for } C_{\mathrm{eq}}\end{array}$ & $1.4 \times 10^{-3}$ \\
\hline$n$ & - & $\begin{array}{c}\text { Thermodynamic } \\
\mathrm{pCO}_{2} \\
\text { dependence }\end{array}$ & 0.316 \\
\hline$\alpha^{*}$ & $\ldots$ & $\begin{array}{c}L \phi \rho_{\mathrm{sf}} A X_{r} \mu \\
\text { (see Section } 2.2 \text { and } \\
\text { below) }\end{array}$ & $3.39 \times 10^{5}$ \\
\hline$L^{*}$ & $\mathrm{~m}$ & Flow path length & 1 \\
\hline$\phi^{*}$ & - & Porosity & 0.1 \\
\hline$\rho_{\mathrm{sf}}{ }^{*}$ & $\mathrm{~kg} \mathrm{~m}^{-3}$ & $\begin{array}{l}\text { Mineral mass to } \\
\text { fluid volume ratio }\end{array}$ & 12728 \\
\hline$A^{*}$ & $\mathrm{~m}^{2} \mathrm{~kg}^{-1}$ & $\begin{array}{l}\text { Specific surface } \\
\text { area }\end{array}$ & 100 \\
\hline$X_{r}^{*}$ & - & $\begin{array}{l}\text { Reactive } \\
\text { mineral conc. } \\
\text { in fresh rock }\end{array}$ & 0.36 \\
\hline
\end{tabular}

Table 1

(Continued)

\begin{tabular}{lccc}
\hline \hline Parameter & Units & Definition & Default Value \\
\hline$\mu^{*}$ & - & Scaling constant & $e^{2}$ \\
\hline$t_{s}^{*}$ & $\mathrm{yr}$ & Soil age & $10^{5}$ \\
\hline$m^{*}$ & $\mathrm{~kg} \mathrm{~mol}^{-1}$ & Mineral molar mass & 0.27 \\
\hline$k_{\text {eff,ref }}{ }^{*}$ & $\begin{array}{c}\mathrm{mol} \mathrm{m}^{-2} \\
\mathrm{yr}^{-1}\end{array}$ & $\begin{array}{c}\text { Reference rate } \\
\text { constant }\end{array}$ & $8.7 \times 10^{-6}$ \\
\hline$\beta$ & $\ldots$ & $\begin{array}{c}\text { Kinetic weathering } \\
\mathrm{pCO}_{2} \text { dependence }\end{array}$ & (e.g., Rimstidt et al. 2012) \\
\hline$T_{e}$ & Kelvin & $\begin{array}{c}\text { Kinetic weathering } \\
\text { temperature } \\
\text { dependence }\end{array}$ & $\begin{array}{c}11.1 \\
\text { (Berner 1994) }\end{array}$ \\
\hline & & &
\end{tabular}

Note. This table lists parameters used in our calculations, their units, their definitions, and the default values they take. A single asterisk $\left(^{*}\right)$ means the default parameter value was drawn from Table S1 of the supplement to Maher \& Chamberlain (2014). For default parameters drawn from other sources, the citation is given in the "Value(s)" column.

and in acidic conditions, reduced $\mathrm{pH}$ often accelerates dissolution. Under neutral-to-acid conditions, the silicate dissolution rate varies with $\mathrm{pH}$ as (e.g., Kump et al. 2000)

$$
\begin{gathered}
r \propto\left[\mathrm{H}^{+}\right]^{n_{\mathrm{H}}}, \\
\log _{10}(r) \propto-n_{\mathrm{H}} \times \mathrm{pH},
\end{gathered}
$$

where $\left[\mathrm{H}^{+}\right]$is the activity or concentration of $\mathrm{H}^{+}$ions and $n_{\mathrm{H}}$ is the reaction order with respect to $\mathrm{H}^{+}$ion activity.

Following Berner (1992), in the limit where there are no influences on the water's $\mathrm{pH}$ except $\mathrm{CO}_{2}$ concentration, the concentration of $\mathrm{H}^{+}$ions is determined by the reaction

$$
\mathrm{CO}_{2}+\mathrm{H}_{2} \mathrm{O} \leftrightarrow \mathrm{H}^{+}+\mathrm{HCO}_{3}^{-},
$$

with an equilibrium constant of

$$
\begin{aligned}
K_{\mathrm{eq}}= & \frac{\left[\mathrm{H}^{+}\right]\left[\mathrm{HCO}_{3}^{-}\right]}{\left[\mathrm{CO}_{2}\right]} \\
= & \frac{\left[\mathrm{H}^{+}\right]^{2}}{\left[\mathrm{CO}_{2}\right]},
\end{aligned}
$$

which implies

$$
\begin{gathered}
{\left[\mathrm{H}^{+}\right] \propto\left[\mathrm{CO}_{2}\right]^{0.5}} \\
\propto \mathrm{pCO}_{2}^{0.5},
\end{gathered}
$$

where $\mathrm{pCO}_{2}$ is the partial pressure of gaseous $\mathrm{CO}_{2}$ in equilibrium with the silicate/water system, the last step following from Henry's Law. Combined with Equation (4), this produces an expression for the $\mathrm{pCO}_{2}$ dependence of silicate dissolution, assuming a water/rock system in equilibrium with gaseous $\mathrm{CO}_{2}$ and without any other influences on $\mathrm{pH}$ :

$$
\begin{aligned}
r & \propto \mathrm{pCO}_{2}^{0.5 \times n_{\mathrm{H}}} \\
& \propto \mathrm{pCO}_{2}^{\beta},
\end{aligned}
$$

where $\beta$ is simply $0.5 \times n_{\mathrm{H}}$. It is important to recognize that the apparently direct dependence of dissolution rate on $\mathrm{CO}_{2}$ is actually mediated by the impact of $\mathrm{CO}_{2}$ on $\mathrm{pH}$ and does not 
reflect a response to the abundance of $\mathrm{CO}_{2}$ as a reactant, implying that other substances that influence $\mathrm{pH}$ in weathering systems could have just as much impact on weathering rates.

Combining the $\mathrm{pCO}_{2}$ dependence and temperature dependence presented above, the kinetically limited dissolution rate of silicate minerals can be represented as

$$
\frac{r}{r_{\text {ref }}}=\exp \left(\frac{T-T_{\text {ref }}}{T_{e}}\right)\left(\frac{\mathrm{pCO}_{2}}{\mathrm{pCO}_{2, \text { ref }}}\right)^{\beta},
$$

where the "ref" subscript refers to a reference value for a given variable. The values of $T_{e}$ and $\beta$, which determine the sensitivity of the reaction rate to changes in $\mathrm{pCO}_{2}$ and temperature, vary considerably for different silicate minerals. We choose the default values listed in Table 1 based on results from laboratory silicate dissolution experiments like those cited after Equation (2). WHAK assume that the global weathering rate is kinetically limited, which would imply that increasing temperature or $\mathrm{pCO}_{2}$ should always increase the flux of cations delivered to the ocean at a given runoff rate. This produces an equation of the form

$$
\frac{W}{W_{\text {ref }}}=\frac{Q}{Q_{\text {ref }}} \exp \left(\frac{T-T_{\text {ref }}}{T_{e}}\right)\left(\frac{\mathrm{pCO}_{2}}{\mathrm{pCO}_{2, \text { ref }}}\right)^{\beta},
$$

where $W$ is the global silicate weathering rate, $Q$ is the global runoff, and the "ref" subscript refers to a reference value for a given variable. This formulation of global weathering adds another temperature dependence on top of the kinetic dependence, as runoff increases with precipitation, which increases with global temperature (e.g., Held \& Soden 2006; O'Gorman \& Schneider 2008). Some formulations of the weathering feedback (e.g., Berner 1994; Pierrehumbert 2010; Abbot et al. 2012) use $\left(Q / Q_{\text {ref }}\right)^{a}$ where $a=0.65$ based on Dunne (1978) and Peters (1984), but Abbot et al. (2012) demonstrated that weathering behavior in the WHAK model is not sensitive to the choice of $a$ for values between 0 and 2, so we will use $a=1$ for simplicity.

Rocky exoplanet climate and habitability studies that include silicate weathering invariably use the WHAK model (e.g., Kite et al. 2011; Abbot et al. 2012; Edson et al. 2012; Kadoya et al. 2014; Kadoya \& Tajika 2014, 2019; Watanabe et al. 2014; Foley 2015; Menou 2015; Abbot 2016; Batalha et al. 2016; Haqq-Misra et al. 2016; Paradise \& Menou 2017; Ramirez 2018; Rushby et al. 2018; Checlair et al. 2019b; Paradise et al. 2020). However, there are orders-of-magnitude discrepancies between the weathering rates predicted by laboratory silicate dissolution experiments and the weathering rates observed in field studies of silicate weathering (Velbel 1993; Malmström et al. 2000; White \& Brantley 2003; Maher et al. 2006). Further, despite a fairly stable climate over the past 3-4 Ga given the long-term trend in solar forcing, Earth has varied between "hothouse," "icehouse," and even snowball states, which could be explained partially by variations in the strength of the negative feedback on Earth's climate (Kump \& Arthur 1997; West et al. 2005; Maher \& Chamberlain 2014).

\subsection{The MAC Model}

The "solute transport model" presented in Maher \& Chamberlain (2014) (MAC) attempts to provide a mechanistic explanation for variations in the strength of the silicate weathering feedback on Earth through time and in different river catchments. The solute transport model is the first model of the silicate weathering feedback to explicitly include a "thermodynamic limit," $C_{\mathrm{eq}}$, on cation concentration in runoff, which emerges due to the control on silicate mineral dissolution by precipitation of secondary minerals like clays (Alekseyev et al. 1997; Maher et al. 2006, 2009; Winnick \& Maher 2018). When silicate dissolution and clay precipitation reach thermodynamic equilibrium, the maximum solute concentration of silicate dissolution products is achieved, maximizing weathering flux for a given runoff. In the MAC framework, for river catchments draining silicate mineral assemblages, the flux of cations delivered to the ocean (and therefore the $\mathrm{CO}_{2}$ sequestration potential) depends on the ratio between the mean fluid travel time through a reactive assemblage $\left(t_{f} \approx L \phi / q\right)$ and the time required for the system to reach $C_{\text {eq }}$, its maximum concentration $\left(t_{\mathrm{eq}} \approx C_{\mathrm{eq}} / r_{n}\right) ; L$ is the reactive flow path length (which may vary with soil thickness (Ferrier \& Kirchner 2008; Gabet \& Mudd 2009), although this depends on the setting where the bulk of weathering takes place (West 2012; Carretier et al. 2018; see also Section S.1 in the supplement to Maher \& Chamberlain 2014), $\phi$ is the effective porosity of the minerals the water is flowing through, $r_{n}$ is the mineral reaction rate, and $q$ is the runoff. $r_{n}=\rho_{\text {sf }} k_{\text {eff }} A X_{r} f_{w}$, where $\rho_{\text {sf }}$ is the ratio of the mass of solid mineral to volume of fluid, $k_{\mathrm{eff}}$ is the dissolution rate constant, $A$ is the specific surface area for the minerals in the assemblage, $X_{r}$ is the fraction of reactive minerals in fresh, unweathered soil/rock, and $f_{w}$ is the fraction of fresh, unweathered soil/rock in the assemblage. $f_{w}=1 /\left(1+m k_{\mathrm{eff}} A t_{s}\right)$, where $m$ is the molar mass of the minerals and $t_{s}$ is the age of the soil. Note that the fraction of unweathered minerals decreases with soil age, and that a higher weathering constant $\left(k_{\text {eff }}\right)$ will lead to a smaller fraction at a given soil age, depleting the soil/rock of reactive minerals. The ratio of mean fluid travel time through an assemblage and mean time to reach thermodynamic equilibrium $\left(t_{f} / t_{e}\right)$ is known as the Damköhler number (Da; Boucher \& Alves 1963); MAC factor out runoff to introduce the "Damköhler coefficient" $\left(D_{w}\right)$ :

$$
D_{w}=\frac{L \phi \rho_{\mathrm{sf}} k_{\mathrm{eff}} A X_{r}}{C_{\mathrm{eq}}\left(1+m k_{\mathrm{eff}} t_{s}\right)}
$$

The weathering rate constant $k_{\text {eff }}$ can be described by an equation with the same functional form as our Equation (13), e.g., $\frac{k_{\text {eff }}}{k_{\text {eff,ref }}}=\exp \left(\frac{T-T_{\text {ref }}}{T_{e}}\right)\left(\frac{\mathrm{pCO}_{2}}{\mathrm{pCO}_{2, \text { ref }}}\right)^{\beta} \cdot D_{w}$ is central to the MAC formulation of weathering; the higher the value of $D_{w}$ for a mineral assemblage being drained by a given flux of runoff, the higher the concentration of cations in the runoff, up to the thermodynamic limit on concentration, $C_{\text {eq }}$. Further, a higher $D_{w}$ value means that an increase in runoff leads to less dilution of solute, allowing for larger changes to weathering rates in response to climate perturbations.

Winnick \& Maher (2018) showed that $C_{\mathrm{eq}}$ should be dependent on $\mathrm{pCO}_{2}$. As discussed in Section 2.1, $\mathrm{pCO}_{2}$ can be an important control on the $\mathrm{pH}$ of water at Earth's surface; this provides a mechanism for changes in $\mathrm{pCO}_{2}$ to change the equilibrium concentrations of reactants and products in mineral dissolution/precipitation reactions. Following Winnick \& Maher (2018), this can be illustrated with a theoretical silicate 
dissolution/clay precipitation reaction:

$$
\begin{aligned}
& d D+2 \mathrm{CO}_{2}(\mathrm{~g})+\mathrm{H}_{2} \mathrm{O} \leftrightarrow s S+2 \mathrm{HCO}_{3}^{-} \\
& +a A+b B+c \mathrm{SiO}_{2}(\mathrm{aq})
\end{aligned}
$$

where $D$ is the dissolving mineral (e.g., plagioclase feldspar), $A$ and $B$, respectively, are the divalent and monovalent weathering products (e.g., $\mathrm{Ca}^{2+}$ and $\mathrm{Na}^{+}$), $S$ is the precipitating secondary mineral (e.g., halloysite), and lower-case letters are stoichiometric coefficients. We can then write the equilibrium constant for the reaction as

$$
\begin{gathered}
K=\frac{\left[\mathrm{HCO}_{3}^{-}\right]^{2}[A]^{a}[B]^{b}[C]^{c}}{\left(\mathrm{pCO}_{2}\right)^{2}} \\
=\frac{z[A]^{2+a+b+c}}{\left(\mathrm{pCO}_{2}\right)^{2}}
\end{gathered}
$$

where $z=\left(\frac{2}{a}\right)^{2}\left(\frac{b}{a}\right)^{b}\left(\frac{c}{a}\right)^{c}$ captures the relative stoichiometric coefficients and the second step assumes that no other reactions influence the concentrations of the reactants. Now, we can write $C_{\text {eq }}$ in terms of $\mathrm{pCO}_{2}, z, K$, and the stoichiometric coefficients:

$$
\begin{aligned}
{[A]=C_{\mathrm{eq}}=} & \left(\frac{K}{z}\right)^{\frac{1}{2+a+b+c}}\left(\mathrm{pCO}_{2}\right)^{\frac{2}{2+a+b+c}} \\
= & \Lambda\left(\mathrm{pCO}_{2}\right)^{n},
\end{aligned}
$$

where $[A]$, the divalent cation concentration, is chosen for $C_{\mathrm{eq}}$ as the delivery of $\mathrm{Ca}^{2+}$ to the oceans by silicate weathering is thought to be the dominant sink for $\mathrm{CO}_{2}$ on geologic timescales through $\mathrm{CaCO}_{3}$ formation and burial (France-Lanord \& Derry 1997; Sun et al. 2016). The temperature dependence of $C_{\text {eq }}$ is weak (see below and Winnick \& Maher 2018), so we will ignore it in this study. This is a powerful formulation because it allows the $\mathrm{pCO}_{2}$ feedback strength to be derived directly from equilibrium chemistry. However, it is also a simplification, as the concentrations of reactants in mineral dissolution and precipitation reactions will be influenced by other, coupled reactions and particularly by the presence of other sources of acidity and alkalinity. We also note that we have only modeled "open-system" weathering in this study, meaning we have assumed that the weathering system is always in equilibrium with the ambient air and is thus continually recharged with $\mathrm{CO}_{2}$ as weathering takes place. In the limit of "closed-system" weathering, weathering takes place in a system that is not recharged with $\mathrm{CO}_{2}$. These two formulations of weathering display different behavior at low $\mathrm{pCO}_{2}$, with $C_{\text {eq }}$ varying linearly with $\mathrm{CO}_{2}$ in the closed-system case. However, Winnick \& Maher (2018) demonstrate that their behavior generally converges at $\mathrm{pCO}_{2} \leqslant 10^{-1}$ bar, and a slightly different functional form of the relationship between $\mathrm{pCO}_{2}$ and $C_{\text {eq }}$ at low $\mathrm{pCO}_{2}$ would not impact the qualitative results of this study.

Still following Winnick \& Maher (2018), we will use the dissolution of plagioclase feldspar (An20) and the precipitation of halloysite as the example reaction to derive default values for the coefficient and exponent in Equation (20):

$$
\begin{aligned}
& 1.66 \mathrm{Ca}_{0.2} \mathrm{Na}_{0.8} \mathrm{Al}_{1.2} \mathrm{Si}_{2.8} \mathrm{O}_{8}+2 \mathrm{CO}_{2}(\mathrm{~g})+3 \mathrm{H}_{2} \mathrm{O} \\
& \leftrightarrow \mathrm{Al}_{2} \mathrm{Si}_{2} \mathrm{O}_{5}(\mathrm{OH})_{4}+0.33 \mathrm{Ca}^{2+}+1.33 \mathrm{Na}^{+}+2 \mathrm{HCO}_{3}^{-} \\
& +2.66 \mathrm{SiO}_{2}(\mathrm{aq}),
\end{aligned}
$$

which gives $n=0.316$ and an equilibrium constant of $K=10^{-13.3}$ at $288 \mathrm{~K}$, implying $\Lambda=0.0014$. As noted above, $K$ is weakly temperature dependent, producing variations in $\Lambda$ with temperature: for the above reaction, $\Lambda(273 \mathrm{~K})=0.0011$ and $\Lambda(373 \mathrm{~K})=0.0033$. This effect provides a weak negative feedback on climate by increasing $C_{\text {eq }}$ with temperature, but simulations we carried out that included this temperature dependence (not shown) differed insignificantly from those that excluded it, so for simplicity we did not include this effect in the simulations shown in this study. It is also important to note that different mineral assemblages can have a large range of values for $n$, and $\Lambda$ can span orders of magnitude for different lithologies (Winnick \& Maher 2018), so we vary these parameters widely in our calculations.

Using Equations (15) and (20) for $D_{w}$ and $C_{\mathrm{eq}}$ and the weathering and solute concentration equations given in MAC, we can derive a version of MAC's weathering equation that includes the impact of temperature and $\mathrm{pCO}_{2}$ on dissolution kinetics and the impact of $\mathrm{pCO}_{2}$ on thermodynamic equilibrium solute concentration (see the supplement to Maher \& Chamberlain 2014 for a full derivation of Equation 22):

$$
\begin{gathered}
C=C_{\mathrm{eq}} \frac{\mu D_{w} / q}{1+\mu D_{w} / q} \\
w=q C_{\mathrm{eq}} \frac{\mu D_{w} / q}{1+\mu D_{w} / q} \\
=\frac{\alpha}{\left[k_{\mathrm{eff}, \mathrm{ref}} \exp \left(\frac{T-T_{\mathrm{ref}}}{T_{e}}\right)\left(\frac{\mathrm{pCO}_{2}}{\mathrm{pCO}_{2, \mathrm{ref}}}\right)^{\beta}\right]^{-1}+m A t_{s}+\alpha\left[\left(q C_{\mathrm{eq}}\right)\right]^{-1}},
\end{gathered}
$$

where $C$ is the solute concentration in runoff, $w$ is weathering per unit surface area, $\alpha=L \phi \rho_{\mathrm{sf}} A X_{r} \mu$ for compactness of notation, and $\mu=e^{2}$ (see supplement to MAC, where $\mu$ is instead called $\tau$ ). Through its thermodynamic impact on $C_{\mathrm{eq}}$ (Equation (20)), $\mathrm{pCO}_{2}$ has a strong effect on the concentration of solute in runoff and thus on the weathering flux and $\mathrm{CO}_{2}$ sink at a given runoff. Figure 1 shows the solute concentrations and weathering fluxes for the weathering assemblage described in the previous paragraph across a wide range of runoffs and $\mathrm{pCO}_{2}$. In the limit where $\frac{1}{m A t_{s}+\alpha /\left(q C_{\mathrm{eq}}\right)} \gg k_{\mathrm{eff}}$, Equation (24) displays the "kinetically limited" weathering behavior assumed in the WHAK model. This happens when $t_{s}$ is small and $q$ or $C_{\text {eq }}$ is large-when relatively fresh, unweathered soil is being flushed out by large enough volumes of runoff to maintain very dilute solute concentrations. When $m A t_{s}$ is large enough relative to other terms not to ignore, $\alpha /\left(q C_{\mathrm{eq}}\right) \sim 0$, and $\left[k_{\text {eff }}\right]^{-1} \sim 0$, e.g., when runoff and the weathering rate constant are both large but $t_{s}$ is nonnegligible so the soil being flushed out by the runoff is already partially weathered, Equation (24) approaches the limit of a constant value of $\alpha / m A t_{s}$, which goes to zero as $t_{s}$ grows very large. Weathering in this limit is 


\section{With MAC weathering, the concentration $(C)$ and flux $\left(w_{m}\right)$ of solute in runoff $(q)$ increase with increasing $\mathrm{pCO}_{2}$; $C$ plateaus at low $q$ while $w_{m}$ plateaus at high $q$}

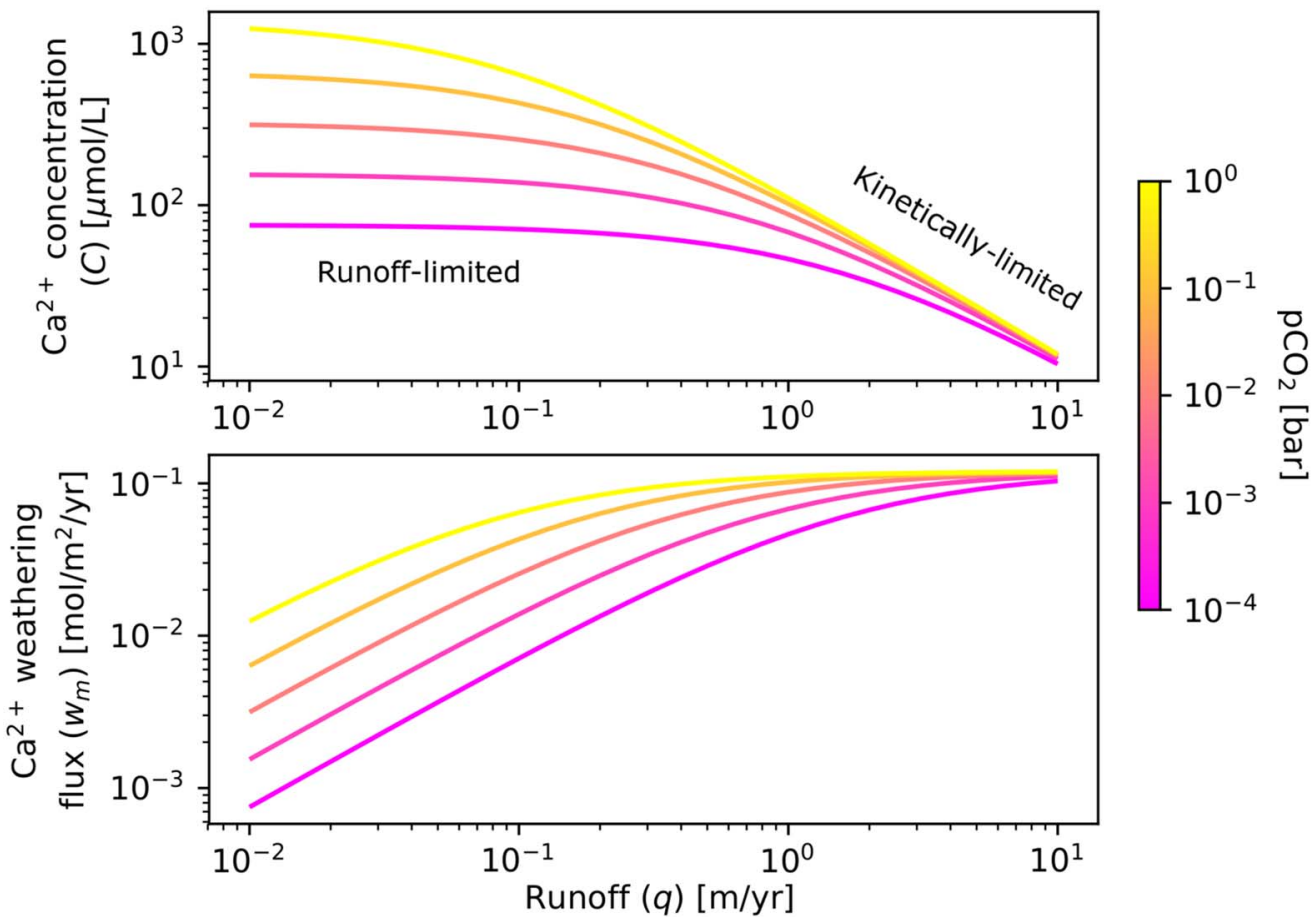

Figure 1. Solute concentrations and weathering fluxes as functions of runoff and $\mathrm{pCO}_{2}$ with our default parameter choices (see Table 1). The top plot shows $\mathrm{Ca}^{2+}$ concentrations (Equation (22)), and the bottom plot shows $\mathrm{Ca}^{2+}$ fluxes to the ocean (Equation (23)). The pinkest curves have a $C_{\mathrm{eq}}$ calculated with pCO $\mathrm{co}_{2}=10^{-4}$ bar (see Equation (20)), and the color of the curves shifts from pink to yellow as $\mathrm{pCO}_{2}$ increases from $10^{-4}$ to $10^{-3}$ to $10^{-2}$ to $10^{-1}$ to $10^{0}$ bar $\mathrm{CO}_{2}$. The regions with runoff limitation and kinetic limitation are labeled as such.

"supply limited," as the weathering rate is dictated by the rate of supply of fresh material to the weathering zone, with a lower $t_{s}$ implying a higher rate of delivery and a less stringent supply limit. When $q C_{\text {eq }} \ll \alpha /\left(m A t_{s}\right)$ and $q C_{\text {eq }} \ll \alpha k_{\text {eff }}$, e.g., when runoff flushes the system out slowly enough relative to other factors that the solute can approach its thermodynamic limit on concentration $C_{\mathrm{eq}}$, the weathering rate varies linearly with runoff. In this regime, the system displays "runoff-limited" (also referred to as "chemostatic") weathering, where the kinetics of silicate dissolution do not influence the weathering rate, and weathering flux is controlled entirely by runoff at a given $\mathrm{pCO}_{2}$.

For context, we will briefly discuss where Earth lies in terms of kinetic versus runoff controls on global weathering flux, using results from studies that apply the MAC framework to interpret proxies and direct weathering measurements. By averaging the annual-mean silica concentrations and fluxes from a data set of large rivers draining granitic lithologies (Gaillardet et al. 1999), Maher \& Chamberlain (2014) estimate that Earth as a whole lies in the transition zone between fully runoff-limited and kinetically limited behavior, with a high degree of variation between individual rivers (see their Figures 2 and 3). von Blanckenburg et al. (2015) combine general circulation model (GCM) simulations and a beryllium weathering proxy to derive a similar result for Earth's weathering regime since the Last Glacial Maximum. Additionally, using nonaveraged solute concentration versus runoff measurements paired with individual estimates of $C_{\mathrm{eq}}$ for rivers draining both granitic and basaltic lithologies, Ibarra et al. (2016) showed that the median concentration-runoff pairs for many individual rivers also lie in the transition regime, though they are on average closer to the runoff limit than the global average estimate by Maher \& Chamberlain (2014). Finally, we note that most of the cases examined in this paper lie in the runofflimited regime or near the transition to kinetic limitation, like Earth.

\section{Model Description and Methods}

Here we describe the models we used to evaluate the consequences of thermodynamic and energetic limits on silicate weathering for the climate stability of rocky land- and ocean-bearing planets in the HZ. We coupled a zerodimensional energy balance model to a zero-dimensional $\mathrm{CO}_{2}$ balance model to simulate the first-order behavior of planets under a variety of conditions. This clearly requires extreme simplification of planetary processes, but this is justified given the lack of observational information about $\mathrm{HZ}$ worlds at present.

The energy balance model equates the global average of absorbed instellation with outgoing longwave radiation (OLR):

$$
\begin{aligned}
S_{\mathrm{avg}}= & \frac{1}{4}(1-a) S \\
& =\mathrm{OLR},
\end{aligned}
$$

where $S_{\text {avg }}$ is the globally averaged absorbed instellation, $S$ is the top-of-atmosphere instellation at the substellar point, $a$ is 
the planetary albedo, and $1 / 4$ is a geometric factor accounting for the fact that a planet's surface area is $4 \times$ the area of its cross section.

By default, our simulations assume a constant planetary albedo $a=0.3$, approximately corresponding to Earth's present-day value (Stephens et al. 2015). We note that a major uncertainty in our study is the exclusion of interactive cloud effects, which strongly impact planetary albedo and energy balance (see Section 5.1.6 for a discussion of this point). We also include a set of simulations with planetary albedo that is a function of surface albedo as well as atmospheric albedo due to Rayleigh scattering by $\mathrm{pCO}_{2}$. This allows us to approximate the impact of atmospheric scattering on the climates of planets orbiting G2 stars like the Sun; the planetary albedos of worlds orbiting $\mathrm{K}$ and $\mathrm{M}$ dwarfs are less affected by Rayleigh scattering, because those classes of stars emit light at longer wavelengths where atmospheres are more strongly absorbing and display weaker scattering (e.g., Kopparapu et al. 2013). The equations for calculating Rayleigh scattering planetary albedo under a two-stream Eddington approximation are (Pierrehumbert 2010)

$$
\begin{gathered}
\tau_{\text {ray }}=0.19513 \frac{\mathrm{pCO}_{2}}{1 \mathrm{bar} \mathrm{CO}_{2}}, \\
a_{a}=\frac{(0.5-0.75 \cos \zeta)\left(1-\exp \left(-\tau_{\text {ray }} / \cos \zeta\right)\right)+0.75 \tau_{\text {ray }}}{1+0.75 \tau_{\text {ray }}} \\
a_{a}^{\prime}=\frac{0.75 \tau_{\text {ray }}}{1+0.75 \tau_{\text {ray }}}, \\
a=1-\frac{\left(1-a_{g}\right)\left(1-a_{a}\right)}{\left(1-a_{g}\right) a_{a}^{\prime}+\left(1-a_{a}^{\prime}\right)}
\end{gathered}
$$

where $\tau_{\text {ray }}$ is the Rayleigh scattering optical depth as a function of $\mathrm{pCO}_{2}$ on a planet with surface gravity equal to Earth's at wavelength $0.5 \mu \mathrm{m}$, which is near the peak of the solar spectrum (drawn from Table 5.2 in Pierrehumbert 2010), $a_{a}$ is the atmospheric albedo due to Rayleigh scattering of incoming solar radiation, $\cos \zeta$ is the cosine of the zenith angle of the star (taken to be $2 / 3$ in this study, based on Cronin 2014), $a_{a}^{\prime}$ is the atmospheric albedo for upward-directed diffuse radiation from the surface, $a_{g}$ is the surface albedo, and the equation for $a$ combines $a_{a}, a_{a}^{\prime}$, and $a_{g}$ to get the total planetary albedo. In the subset of experiments that includes this representation of Rayleigh scattering, we vary $a_{g}$ from 0.1 to 0.3 , covering a range of plausible surface albedos that Earth may have displayed throughout its history as cloud and continental coverage evolved (Rosing et al. 2010).

OLR is calculated using a polynomial fit presented in Kadoya \& Tajika (2019) that approximates the output of the 1D radiative-convective model used in Kopparapu et al. (2013) with 1 bar $\mathrm{N}_{2}$ and saturated $\mathrm{H}_{2} \mathrm{O}$ for temperatures between 150 and $350 \mathrm{~K}$, and $\mathrm{pCO}_{2}$ between $10^{-5}$ and 10 bar:

$$
\begin{aligned}
& \operatorname{OLR}\left(T, \mathrm{pCO}_{2}\right)=I_{0}+\boldsymbol{T B} \Upsilon^{t},
\end{aligned}
$$

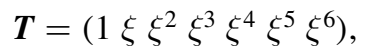

$$
\begin{aligned}
& \Upsilon \mathbf{\Upsilon}=\left(1 v v^{2} v^{3} v^{4}\right),
\end{aligned}
$$

where $T$ is the surface temperature in Kelvin, $\mathrm{pCO}_{2}$ is the partial pressure of $\mathrm{CO}_{2}$ in bars, $I_{0}=-3.1 \mathrm{~W} \mathrm{~m}^{-2}$, and $\xi=$ $0.01 \times(T-250)$. For $\mathrm{pCO}_{2}<1$ bar,

$$
\boldsymbol{B}=\left[\begin{array}{llllll}
87.8373 & 311.289 & 504.408 & 422.929 & 134.611 \\
54.9102 & 677.741 & 1440.63 & 1467.04 & 543.371 \\
24.7875 & 31.3614 & 364.617 & 747.352 & 395.401 \\
75.8917 & 816.426 & 1565.03 & 1453.73 & 476.475 \\
43.0076 & 339.957 & 996.723 & 1361.41 & 612.967 \\
31.4994 & 261.362 & 395.106 & 261.600 & 36.6589 \\
28.8846 & 174.942 & 378.436 & 445.878 & 178.948
\end{array}\right] .
$$

For $10 \mathrm{bar}>\mathrm{pCO}_{2}>1$ bar,

$$
\boldsymbol{B}=\left[\begin{array}{llllll}
87.8373 & 52.1056 & 35.2800 & 1.64935 & 3.42858 \\
54.9102 & 49.6404 & 93.8576 & 130.671 & 41.1725 \\
24.7875 & 94.7348 & 252.996 & 171.685 & 34.7665 \\
75.8917 & 180.679 & 385.989 & 344.020 & 101.455 \\
43.0076 & 327.589 & 523.212 & 351.086 & 81.0478 \\
31.4994 & 235.321 & 462.453 & 346.483 & 90.0657 \\
28.8846 & 284.233 & 469.600 & 311.854 & 72.4874
\end{array}\right] .
$$

This simple parameterization of OLR allows us to perform rapid simulations across a wide range of parameters, with a maximum absolute error of $3.3 \mathrm{~W} \mathrm{~m}^{-2}$ and an average absolute error of $0.6 \mathrm{~W} \mathrm{~m}^{-2}$ in the range of temperatures and $\mathrm{pCO}_{2}$ mentioned above (Kadoya \& Tajika 2019). The main drawback in using this parameterization is that we cannot evaluate climates at $\mathrm{pCO}_{2}$ above 10 bar or with varying $\mathrm{N}_{2}$ abundance.

The $\mathrm{CO}_{2}$ balance model sets $\mathrm{CO}_{2}$ outgassing from volcanism equal to $\mathrm{CO}_{2}$ consumption from weathering:

$$
v=w,
$$

where $v=V /\left(4 \pi R_{\text {planet }}^{2}\right)$ is the total volcanic outgassing of $\mathrm{CO}_{2}(V)$ divided by the planetary surface area, $R_{\text {planet }}$ is the radius of the planet, and $w$ is the $\mathrm{CO}_{2}$ consumption by weathering per unit planetary surface area. To compare the behavior of planets with the WHAK and MAC weathering models, we run simulations with each formulation:

$$
w_{w}=w_{w, \text { ref }} \frac{\gamma p}{\gamma_{\text {ref }} p_{\text {ref }}} \exp \left(\frac{T-T_{\text {ref }}}{T_{e}}\right)\left(\frac{\mathrm{pCO}_{2}}{\mathrm{pCO}_{2, \text { ref }}}\right)^{\beta},
$$

$$
w_{m}=\gamma \frac{\alpha}{\left[k_{\text {eff,ref }} \exp \left(\frac{T-T_{\text {ref }}}{T_{e}}\right)\left(\frac{\mathrm{pCO}_{2}}{\mathrm{pCO}_{2, \text { ref }}}\right)^{\beta}\right]^{-1}+m A t_{s}+\alpha\left[q \Lambda\left(\mathrm{pCO}_{2}\right)^{n}\right]^{-1}},
$$


where $w_{w}$ is the weathering per unit planetary area using the WHAK model; $\gamma$ is the global land fraction, which is included to account for the fact that continental silicate weathering only happens on continents; $p$ is the global average precipitation per unit area; and $w_{m}$ is the weathering per unit planetary area using the MAC model. Crucially, the impact of the kinetic dissolution rate and its dependence on temperature and $\mathrm{pCO}_{2}$ are greatly weakened in Equation (40). This is because the increased dissolution of silicates also depletes soils and rocks of their reactive components more completely for a given soil age, reducing the reactivity of the assemblage and counteracting the increase in dissolution rate. However, $\mathrm{pCO}_{2}$ still has a powerful impact on silicate weathering through its control of the thermodynamic equilibrium concentration of solute in runoff (Equation (20)).

We take the runoff $q$ to be a linear function of precipitation $p$, which is itself taken to be a linear function of temperature $T$ (e.g., Held \& Soden 2006; O'Gorman \& Schneider 2008):

$$
\begin{gathered}
q=\Gamma \times p, \\
p=p_{\text {ref }}\left(1+\epsilon\left(T-T_{\text {ref }}\right)\right),
\end{gathered}
$$

where $\Gamma$ is the proportionality constant between precipitation and runoff and $\epsilon$ is the fractional change in global precipitation per Kelvin deviation from a reference surface temperature.

We also include the upper limit on precipitation noted by Pierrehumbert (2002) and O'Gorman \& Schneider (2008). In steady state, global precipitation is equal to global evaporation. Global evaporation is ultimately driven by instellation, which provides the energy required for water to transition from liquid to gas (the latent heat of vaporization). If there is not enough instellation to make up for the cooling caused by a given amount of evaporation, then a planet cannot sustain that level of precipitation. This implies a maximum global precipitation given by

$$
\begin{gathered}
p_{\lim }=\frac{S_{\mathrm{avg}}}{L(T)}, \\
L(T)=1.918 \times 10^{9}\left(\frac{T}{T-33.91}\right)^{2},
\end{gathered}
$$

where $p_{\text {lim }}$ is the maximum precipitation sustainable on a planet at a given instellation and $L(T)$ is the latent heat of vaporization for water in $\mathrm{J} \mathrm{m}^{-3}$, converted from $L$ in $\mathrm{J} \mathrm{kg}^{-1}$ given by the Henderson-Sellers equation (Henderson-Sellers 1984) with multiplication by water's density $1000 \mathrm{~kg} \mathrm{~m}^{-3}$.

More speculatively, we propose that the actual value of $p_{\text {lim }}$ at a given instellation may be lower than that given by Equation (43) for planets with land, as stellar energy absorbed by continents can only recycle water that ultimately evaporated from the ocean (note that the validity of Equation (43) was only demonstrated in the case of a fully ocean-covered planet by O'Gorman \& Schneider 2008). Although the influence of a given amount of land area on the energetic precipitation limit should depend on the latitude of the land mass (e.g., a continent will intercept many more photons if it straddles the equator or substellar point than if it sits on a pole or at the terminator) and the efficiency of the atmosphere at moving energy from the land surface to the ocean surface, we suggest an approximate, first-order scaling of $p_{\text {lim }}$ with global ocean fraction $(1-\gamma)$, such that Equation (43) represents $p_{\text {lim }}$ in the $100 \%$ ocean coverage limit:

$$
p_{\text {lim,land }}=(1-\gamma) \frac{S_{\mathrm{avg}}}{L(T)} \text {, }
$$

where $p_{\text {lim,land }}$ is the energetic limit on precipitation for planets with a nonzero surface land fraction. Because we have not yet demonstrated the validity of Equation (45) explicitly with GCM simulations (a subject for future work), we use Equation (43) for the energetic limit in most calculations, but we also carry out a set of experiments with Equation (45) to examine the impact of the proposed scaling.

We search for equilibrium $\mathrm{pCO}_{2}$ and $T$ for planets at $S$ from 1400 to $473 \mathrm{~W} \mathrm{~m}^{-2}$, the outer edge of the classical HZ for an Earth-size planet orbiting a $G$ star according to Kopparapu et al. (2013), defined by the "maximum greenhouse" limit where Rayleigh scattering from $\mathrm{CO}_{2}$ accumulation begins to outweigh $\mathrm{CO}_{2}$ 's warming effect. Note that we do not have a representation of Rayleigh scattering in our default model, so we take the value of the outer edge given in Kopparapu et al. (2013), though we also explore the impact of a Rayleigh scattering parameterization in a separate set of simulations. To find equilibrium values, we solve for $\mathrm{pCO}_{2}$ and $T$ where $v=w$ and $S_{\text {avg }}=$ OLR simultaneously. Using this procedure to find steady-state climates, we compare the behavior of planets with weathering described by the MAC formulation against that of planets under the WHAK formulation and vary parameters across a range of values to examine the sensitivity of our models. In particular, we examine the $\mathrm{pCO}_{2}$ and temperatures of our simulations as a function of instellation, and we calculate the "effective outer edge of the HZ" as the instellation at which the temperature of a simulation with a given set of parameters drops below freezing (while acknowledging that planets may be able to reach global average temperatures somewhat below freezing before entering a "hard snowball state"; e.g., Abbot et al. 2011; Yang et al. 2012). In the next section, we present the results of these calculations.

\section{Results}

We begin by comparing the simulations with the WHAK formulation against the simulations with the MAC formulation. The different weathering models lead to qualitative differences in weathering behavior, planetary climate, and the effective outer edge of the HZ. Then, we examine the impact of varying the parameters that represent hydrology, weathering thermodynamics, surface properties, and albedo in our model of the MAC formulation of weathering. The effective outer edge of the HZ is quite sensitive to all of these sets of parameters. This makes it difficult to predict what fraction of rocky, oceanbearing planets in the $\mathrm{HZ}$ we should expect to find in a temperate state instead of a snowball or a moist greenhouse, even if we ignore the strong possibility that other greenhouse gases or $\mathrm{CO}_{2}$ cycling mechanisms will be present on putatively Earth-like planets. Finally, we examine the impact of the energetic limit on precipitation set by planetary instellation (see Equations (43) and (45)), which we find fundamentally changes the functioning of the silicate weathering feedback under some circumstances by decoupling planetary temperature and global runoff. 
MAC-style weathering (solid curves) is more sensitive to land fraction $(\gamma)$ than WHAK-style weathering (dashed curves)

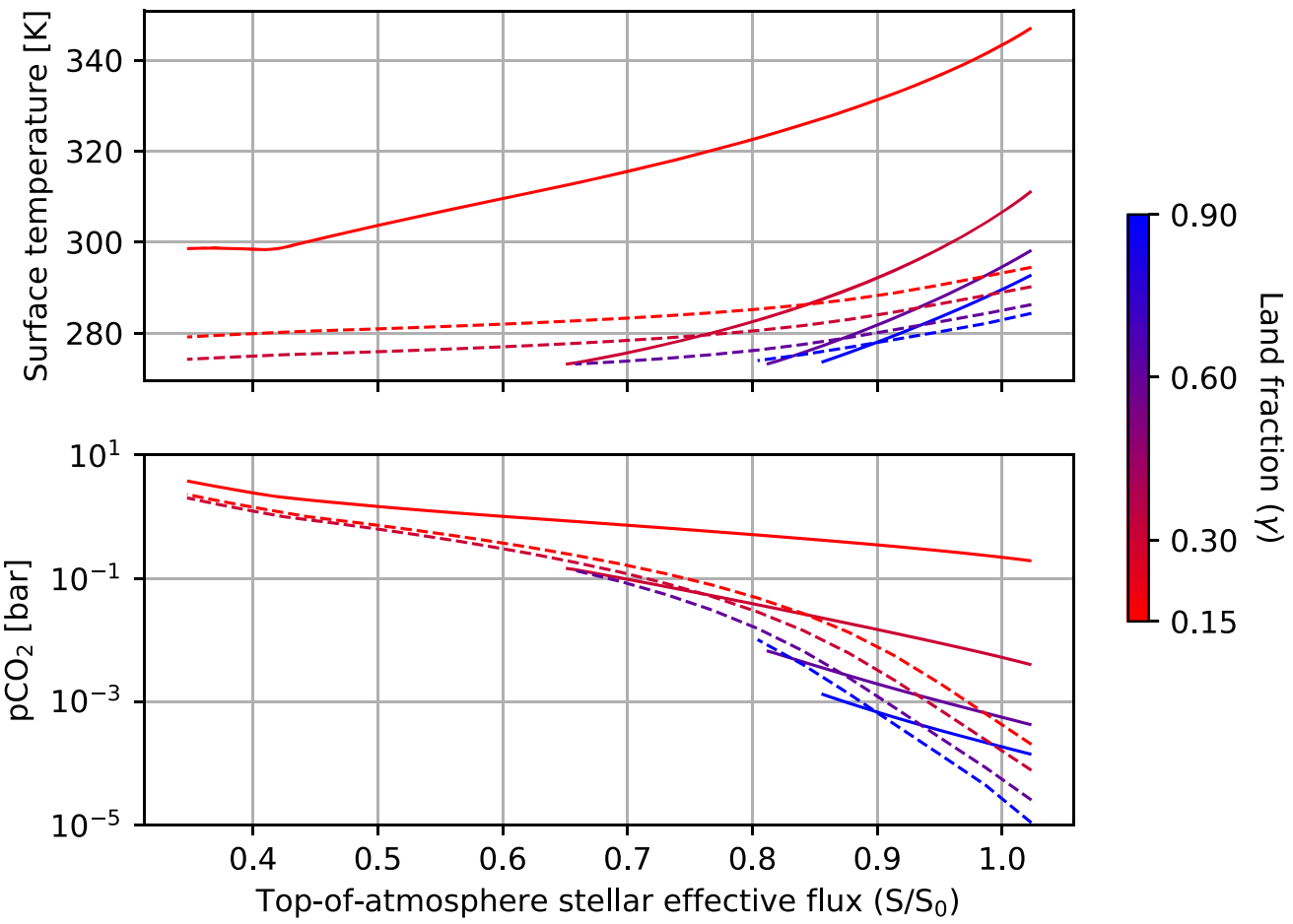

Figure 2. Comparison of instellation vs. temperature and $\mathrm{pCO}_{2}$ for $\mathrm{WHAK}$ and $\mathrm{MAC}$ simulations at varying land fractions. The top plot shows top-of-atmosphere stellar effective flux $\left(S / S_{0}\right.$, where $\left.S_{0}=1368 \mathrm{~W} \mathrm{~m}^{-2}\right)$ vs. temperature. The bottom plot shows TOA stellar effective flux vs. pCO . Solid curves use the MAC formulation of weathering, and dashed curves use the WHAK formulation. The reddest curves have land fraction $(\gamma)=0.15$. $\gamma=0.3$ curves are red-purple, $\gamma=0.6$ curves are blue-purple, and $\gamma=0.9$ curves are blue.

\subsection{WHAK versus MAC}

\subsubsection{Sensitivity to Land Fraction and $\mathrm{CO}_{2}$ Outgassing Rate}

As demonstrated in Abbot et al. (2012) and Foley (2015), weathering behavior with the WHAK model is fairly insensitive to planetary land fraction; in contrast, MAC planet climates are considerably more sensitive to land fraction, particularly at $\gamma<0.3$ (see Figure 2 and the leftmost column in Figure 3). The MAC simulation with $\gamma=0.15$ is $30-40 \mathrm{~K}$ warmer than the simulation with $\gamma=0.3$ at all instellations, and with $\gamma<0.15$, we failed to find stable solutions within the temperature and $\mathrm{pCO}_{2}$ range of the OLR parameterization we are using, though stable climates conceivably exist with $\mathrm{pCO}_{2}>10 \mathrm{bar}$ and/or $T>350 \mathrm{~K}$. Also, other combinations of parameters would lead to different values in either direction for the minimum $\gamma$, so $\gamma=0.15$ is not a hard minimum on the land fraction a planet needs to stabilize its climate via silicate weathering. Interestingly, Figure 2 shows that the temperature of the $\gamma=0.15$ case stops decreasing with instellation when $S / S_{0}$ falls below $\sim 0.4$ (see the red curve in the top panel of Figure 2). This is because that set of simulations hits the energetic limit on precipitation at that instellation (see Section 4.3 for further analysis of this phenomenon). Because $v=w$ and $\gamma$ is a multiplicative factor on the front of the equations for $w_{w}$ and $w_{m}$ (Equations (39) and (40)), a fractional change in $\gamma$ at constant $v$ has the same effect as multiplying $v$ by the reciprocal of that fraction while holding $\gamma$ constant. So, planets under the MAC formulation are also sensitive to increases in $\mathrm{CO}_{2}$ outgassing rates, as reducing $\gamma$ by a factor of 2 from 0.3 to 0.15 is equivalent to multiplying volcanic outgassing by 2 while holding land fraction constant.

The difference in land fraction and outgassing sensitivity between MAC and WHAK planets is due to the lack of weathering sensitivity to dissolution kinetics in the MAC planets. Under decreased land fraction (or increased outgassing), more weathering must happen per unit land area to balance outgassing. Without temperature-dependent changes in solute concentration via changes in dissolution kinetics, MAC planets are forced to compensate for changes in land fraction or outgassing through changes to precipitation rate (which responds to temperature) and maximum solute concentration $C_{\text {eq }}$ (which responds to $\mathrm{pCO}_{2}$ ). On WHAK planets, the solute concentration can increase or decrease without limit in response to both temperature and $\mathrm{pCO}_{2}$ due to changes in the kinetic silicate dissolution rate. This allows for greater changes in concentration to bolster changes in runoff in response to altered land area or outgassing rate on WHAK planets. This means smaller changes in precipitation are necessary to alter weathering fluxes to balance land fraction and outgassing changes. Smaller precipitation changes imply smaller temperature changes.

\subsubsection{Sensitivity to Parameters Controlling Silicate Dissolution Kinetics}

In agreement with Abbot (2016), the climates of WHAK planets as a function of instellation in our models are sensitive to the kinetic $\mathrm{pCO}_{2}$ dependence $(\beta$; dashed curves in the middle column in Figure 3$)$ and temperature dependence $\left(T_{e}\right.$; dashed 
Temperature, $\mathrm{PCO}_{2}$, and $\mathrm{HZ}$ outer edge of $\mathrm{MAC}$ simulations (solid)

are more sensitive to land fraction but less sensitive to silicate dissolution kinetics than WHAK (dashed)
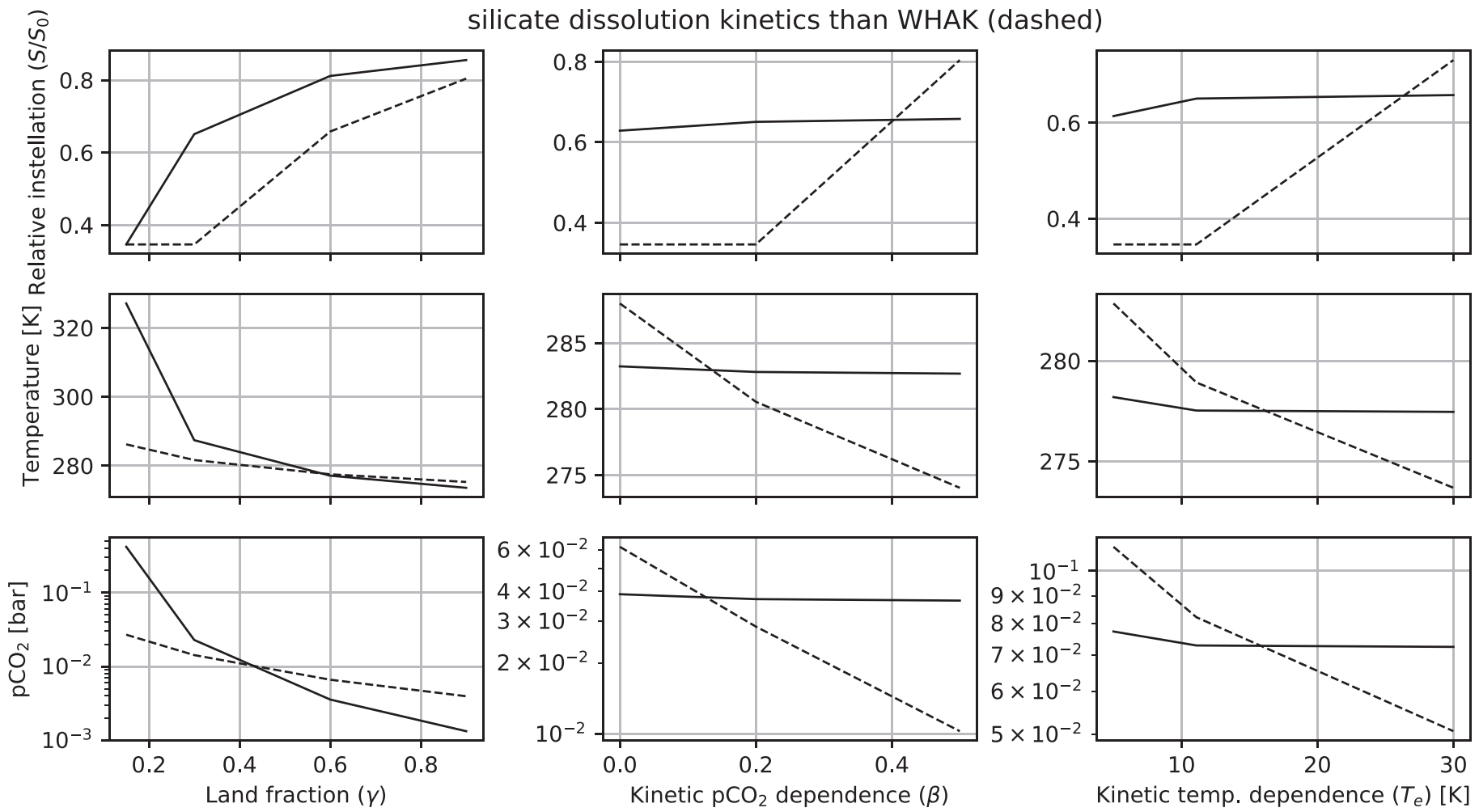

Figure 3. Comparison of the sensitivity to land fraction and silicate dissolution kinetics of WHAK simulations and MAC simulations. Dashed curves show results for WHAK simulations, solid curves show results for MAC simulations. The top row shows the relative instellation $\left(S / S_{0}\right.$, where $\left.S_{0}=1368 \mathrm{~W} \mathrm{~m}{ }^{-2}\right)$ of the outer edge of the HZ, defined as the instellation where planetary temperature drops below freezing with a given set of weathering parameters. If the calculated outer edge is below the classical outer edge for Earth as defined in Kopparapu et al. (2013), and the outer edge is set to the classical outer edge $\left(S / S_{0}=1 / 1.7^{2}=0.346\right)$. The middle row shows the temperature for each simulation at the highest $\mathrm{HZ}$ outer edge instellation of the group of simulations in that column. The bottom row shows the pCO ${ }_{2}$ for each simulation at the highest $\mathrm{HZ}$ outer edge instellation of the group of simulations in that column. The leftmost column shows simulations under varying land fractions $(\gamma)$, the center column shows simulations under varying kinetic $\mathrm{pCO}_{2}$ dependences $(\beta)$, and the right column shows simulations under varying kinetic temperature dependences $\left(T_{e}\right)$. Any parameters not being varied take their default value.

curves in the rightmost column in Figure 3) in Equation (39). For high values of $\beta$, weathering is greatly accelerated by high $\mathrm{pCO}_{2}$, which increases $\mathrm{CO}_{2}$ drawdown and induces cooling at low instellations where high $\mathrm{pCO}_{2}$ is required for habitability. This means that lower $\beta$ values translate to better climate control as a function of instellation in the WHAK formulation, with the limit of a perfect thermostat at $\beta=0$, other things being equal (Pierrehumbert 2010; Ramirez 2017). Lower $T_{e}$ values also translate to more effective climate control, as weathering rates respond more strongly to a given change in temperature. In contrast to the results for the WHAK formulation, the climates of MAC planets with our default parameter choices show almost no sensitivity to $\beta$ and $T_{e}$ (solid curves in the middle and rightmost columns of Figure 3 ). This is because increased kinetic dissolution rates (e.g., increased $k_{\text {eff }}$ ) tend to deplete mineral assemblages of reactive components, reducing the impact of kinetics on steady-state weathering intensity. Kinetics only influences the steady-state weathering flux when the dissolution rate is very slow relative to the rate at which the weathering system is flushed out by runoff. Our MAC simulations are far from the condition where the kinetic dissolution rate determines weathering flux (see final paragraph in Section 2.2). Instead, weathering flux is determined by the interplay between runoff, cation concentration, and $\mathrm{pCO}_{2}$ governed by the thermodynamics of silicate dissolution and clay precipitation (see Figure 1 and Section 2.2).

\subsection{Sensitivity to Hydrology, Thermodynamic $\mathrm{pCO}_{2}$ Dependence, Surface Properties, and Rayleigh Scattering Albedo}

The climates of planets under the MAC formulation are very sensitive to hydrology, the thermodynamics of silicate dissolution and clay precipitation, and surface properties in weathering assemblages because these factors combine to control the concentration and flux of cations to the ocean. Rayleigh scattering albedo can also have a strong effect on the behavior of planets near the outer edges of the HZs of G stars because of both its direct radiative effects and its effects on weathering arising from energetic limits on precipitation and runoff.

\subsubsection{Hydrology}

The hydrologic cycle is parameterized roughly in our model by making runoff linearly dependent on precipitation and precipitation linearly dependent on temperature.

Increasing $\Gamma$, the fraction of precipitation converted to runoff, reduces temperature and $\mathrm{pCO}_{2}$ and moves the effective outer edge of the HZ closer to the star (see the leftmost column of Figure 4). This is because a greater quantity of runoff per 
Planetary temperature, $\mathrm{pCO}_{2}$, and $\mathrm{HZ}$ outer edge are very sensitive to
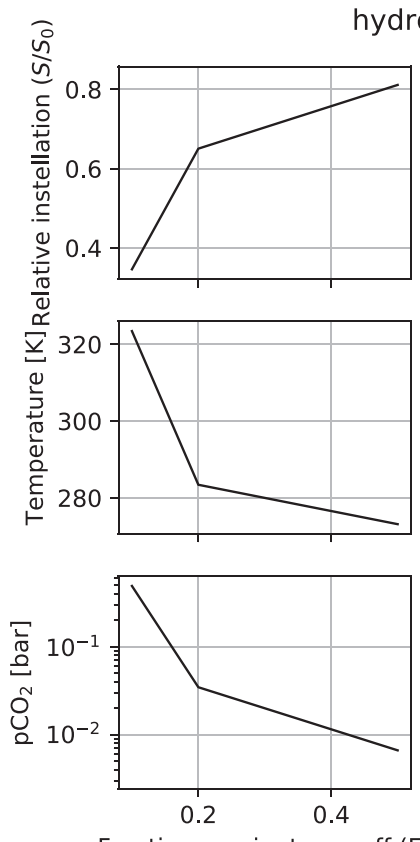

Fraction precip. to runoff $(\Gamma)$ ydrology, weathering thermodynamics, and surface properties with MAC weathering
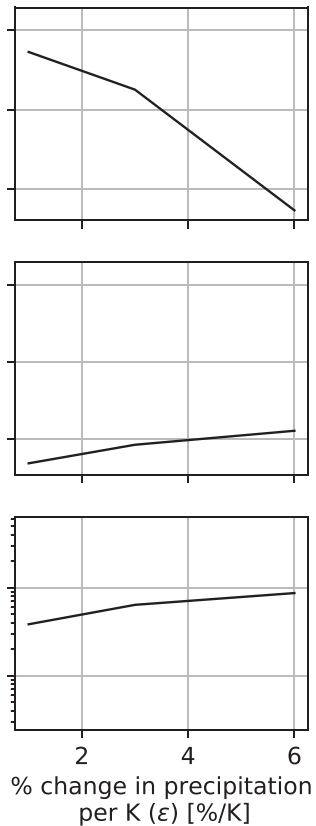
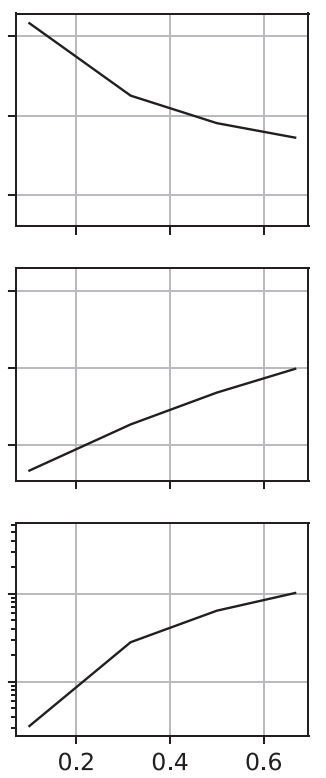

Thermodynamic $\mathrm{pCO}_{2}$ dependence $(n)$
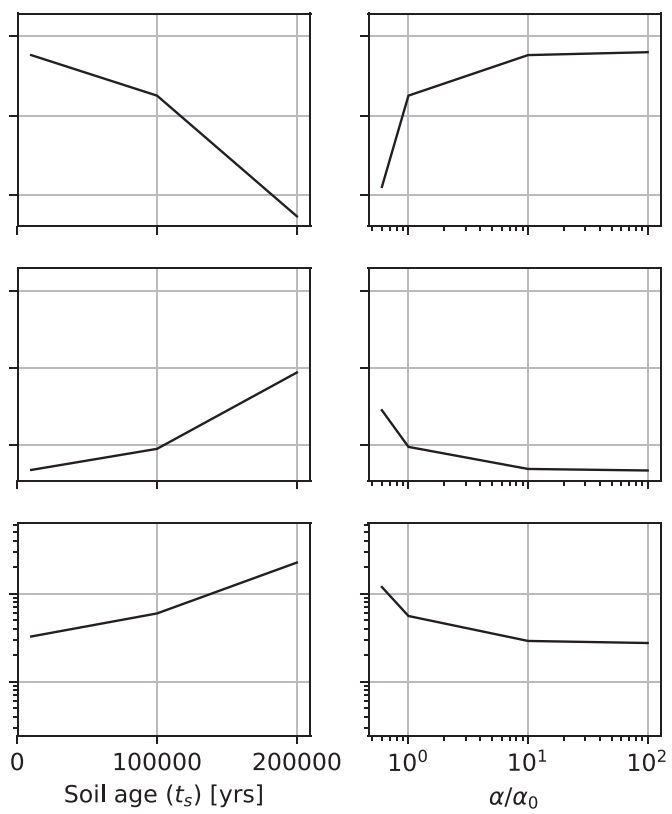

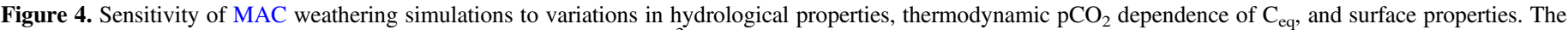

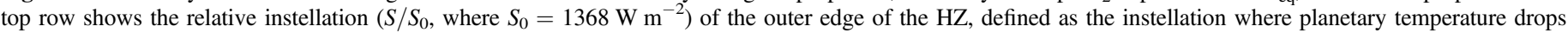

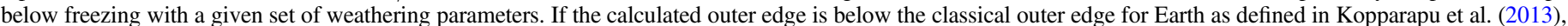

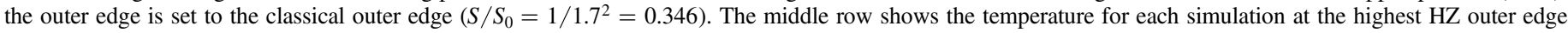

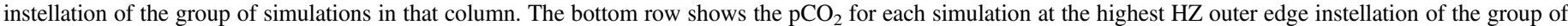

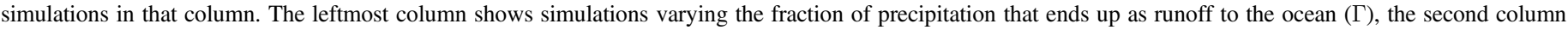

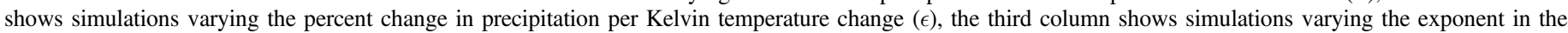

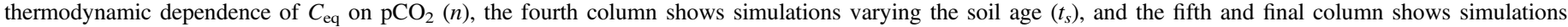

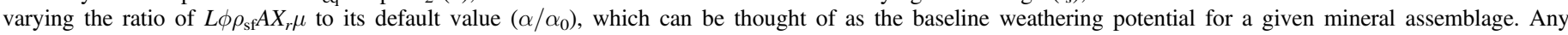
parameters not being varied take their default value.

unit precipitation leads to a larger weathering flux at a given temperature and $\mathrm{pCO}_{2}$, allowing for a given outgassing rate to be balanced with less rain at colder temperatures. This effect is also illustrated in the bottom panel of Figure 1: a given weathering flux can be achieved at successively lower $\mathrm{pCO}_{2}$ by increasing runoff (moving rightward in the plot). Increasing $\epsilon$, the fractional change in precipitation per unit Kelvin, moves the effective outer edge of the $\mathrm{HZ}$ away from the star quite strongly, but does not have as much impact on temperature and $\mathrm{pCO}_{2}$ (see second column in Figure 4). $\epsilon$ governs the strength of the temperature dependence of the silicate weathering feedback in the MAC model, as it controls how much the global weathering flux changes in response to temperature changes. Increasing $\epsilon$ makes the weathering flux more sensitive to temperature, which allows a planet with a given set of parameters to maintain balance against $\mathrm{CO}_{2}$ outgassing out to lower instellations before hitting the freezing point.

\subsubsection{Thermodynamic Control of $\mathrm{C}_{e q}$}

Increasing the thermodynamic $\mathrm{pCO}_{2}$ dependence of $C_{\mathrm{eq}}$ in isolation ( $n$ in Equations (20) and (40)) at a given $\mathrm{pCO}_{2}$ below 1 bar tends to reduce $C_{\mathrm{eq}}$, which reduces weathering flux, necessitating a larger $\mathrm{pCO}_{2}$ to balance outgassing. So, increasing $n$ tends to move the outer edge of the HZ farther from the star and increase temperatures and $\mathrm{pCO}_{2}$ (see the middle column of Figure 4).

Increasing the thermodynamic coefficient $\Lambda$ tends to increase $C_{\text {eq }}$, which allows the concentration of solute in runoff to reach higher levels and therefore allows for a larger maximum weathering flux at a given runoff rate. This means increases to $\Lambda$ cool planets, moving the effective outer edge of the $\mathrm{HZ}$ closer to the star (Figure 5).

\subsubsection{Surface Properties}

The physical properties of the surface of a planet have important impacts on its weathering behavior which have not been considered in previous studies of exoplanet weathering.

Increasing soil age $\left(t_{s}\right)$ tends to move the effective outer edge of the $\mathrm{HZ}$ farther from the star and increase planetary temperature and $\mathrm{pCO}_{2}$ (see the second-from-right column in Figure 4). This is because older soils are more depleted of weatherable minerals. This increases the time necessary for water moving through a mineral assemblage to reach thermodynamic equilibrium between silicate dissolution and clay precipitation ( $t_{\mathrm{eq}}$; see Section 2.2), which decreases solute concentration and increases the quantity of runoff necessary to generate a large enough weathering flux to balance outgassing. At high-enough $t_{s}$, reactive minerals are so depleted that the negative feedback between weathering and climate is lost, as weathering rates are dictated by the supply of reactive minerals to the assemblage ("supply-limited" weathering (West et al. 2005; Foley 2015) — not shown, as no equilibrium climate is possible in this regime with our models). The $t_{s}$ where supply limitation sets in varies depending on the values taken by other parameters. 


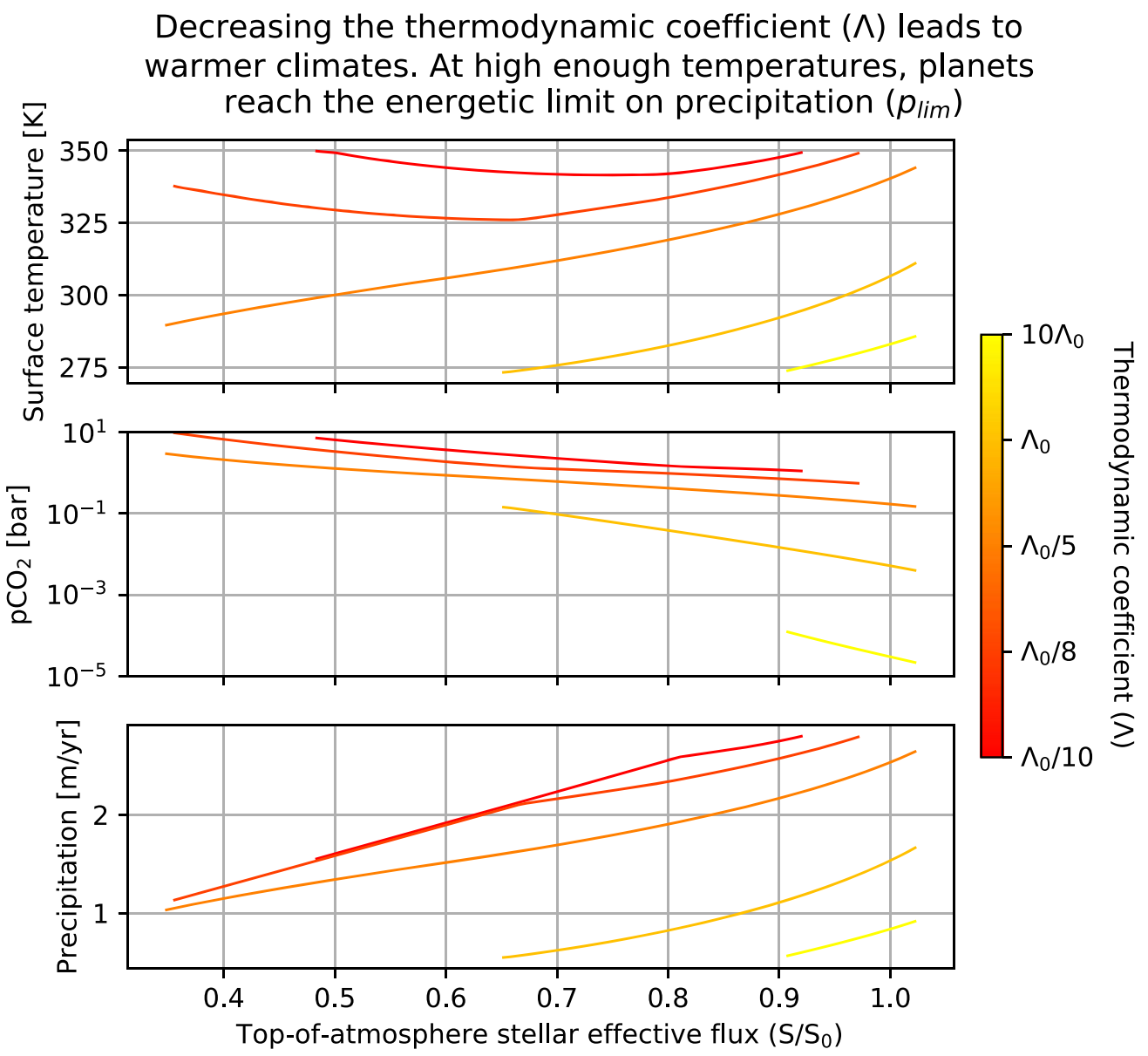

Figure 5. Comparison of temperature, $\mathrm{pCO}_{2}$, and precipitation vs. instellation for simulations varying thermodynamic coefficient for $C_{\mathrm{eq}}$ 's dependence on pCO $\mathrm{O}_{2}(\Lambda)$. The top plot shows instellation vs. temperature $(T)$, the center plot shows instellation vs. $\mathrm{pCO}_{2}$, and the bottom plot shows instellation vs. precipitation. The reddest curves use $\Lambda=\Lambda_{0} / 10$ (where $\Lambda_{0}=1.4 \times 10^{-3}$ ), and the curves become yellower as $\Lambda$ is increased through $\Lambda_{0} / 8, \Lambda_{0} / 5, \Lambda_{0}$, and $10 \Lambda_{0}$. Parameters not being varied take their default values.

$\alpha$ is the product of several different parameters: reactive flow path length $(L)$, porosity of the mineral assemblage being weathered $(\rho)$, the ratio of mineral mass to fluid volume $\left(\rho_{\mathrm{sf}}\right)$, the specific surface area of minerals being weathered $(A)$, the concentration of reactive minerals in fresh, unweathered bedrock $\left(X_{r}\right)$, and a scaling constant that brings the theoretical scaling of solute concentration in line with reactive transport modeling ( $\mu$; see the supplement to Maher \& Chamberlain 2014). Increasing any of these parameters brings water flowing through a mineral assemblage closer to thermodynamic equilibrium at a given runoff flux, so $\alpha$ might be thought of as a set of physical properties that determines the baseline weathering potential for a mineral assemblage (an aspect of "weatherability"; e.g., Kump \& Arthur 1997). Moving the solute concentration closer to its thermodynamic limit $C_{\text {eq }}$ at constant runoff increases the weathering flux (see Figure 1), drawing down $\mathrm{CO}_{2}$, reducing temperature, and moving the effective outer edge of the HZ closer to the star (see the rightmost column in Figure 4).

\subsubsection{Rayleigh Scattering Albedo}

We use Equation (30) to estimate the effect on climate of planetary albedo (a) variations due to Rayleigh scattering at $0.5 \mu \mathrm{m}$ by $\mathrm{CO}_{2}$ for Earth-mass planets with a range of surface albedos orbiting Sun-like stars, and we compare the simulations with interactive albedo to simulations with a constant planetary albedo equal to the reflectivity of the surface/clouds (see Figure 6). Our approach to estimating Rayleigh scattering albedo likely overestimates its importance to planetary energy balance, as atmospheric near-infrared absorption should also increase with $\mathrm{pCO}_{2}$, so these simple calculations represent a conservative upper bound on the strength of Rayleigh scattering. We used a reduced thermodynamic coefficient of $\Lambda=\Lambda_{0} / 5$ to decrease $C_{\mathrm{eq}}$ and force the simulations to accumulate large $\mathrm{pCO}_{2}$ so that the $\mathrm{CO}_{2}$-induced Rayleigh scattering effect would be clearer. We should also note that any change to a parameter that increases $\mathrm{pCO}_{2}$ (e.g., an increase in soil age) would have the same effect on Rayleigh scattering as the reduction in $\Lambda$ that we use; there is no specific relationship between $\Lambda$ and Rayleigh scattering. At low $\mathrm{pCO}_{2}$, the impact of $\mathrm{CO}_{2}$-induced Rayleigh scattering is negligible, leading to changes in albedo of order $1 \%$; under these circumstances, planetary albedo is dominated by the prescribed albedo of the surface (which represents the combined surface albedo, cloud albedo, and albedo of the non- $\mathrm{CO}_{2}$ part of the atmosphere). But, as expected, as $\mathrm{pCO}_{2}$ grows with decreasing instellation (middle panel in Figure 6), elevated Rayleigh scattering leads to increased albedo (solid curves in bottom panel of Figure 6), which in turn cools a simulation relative to the corresponding simulation without Rayleigh scattering (compare solid and dashed curves in the top panel of 


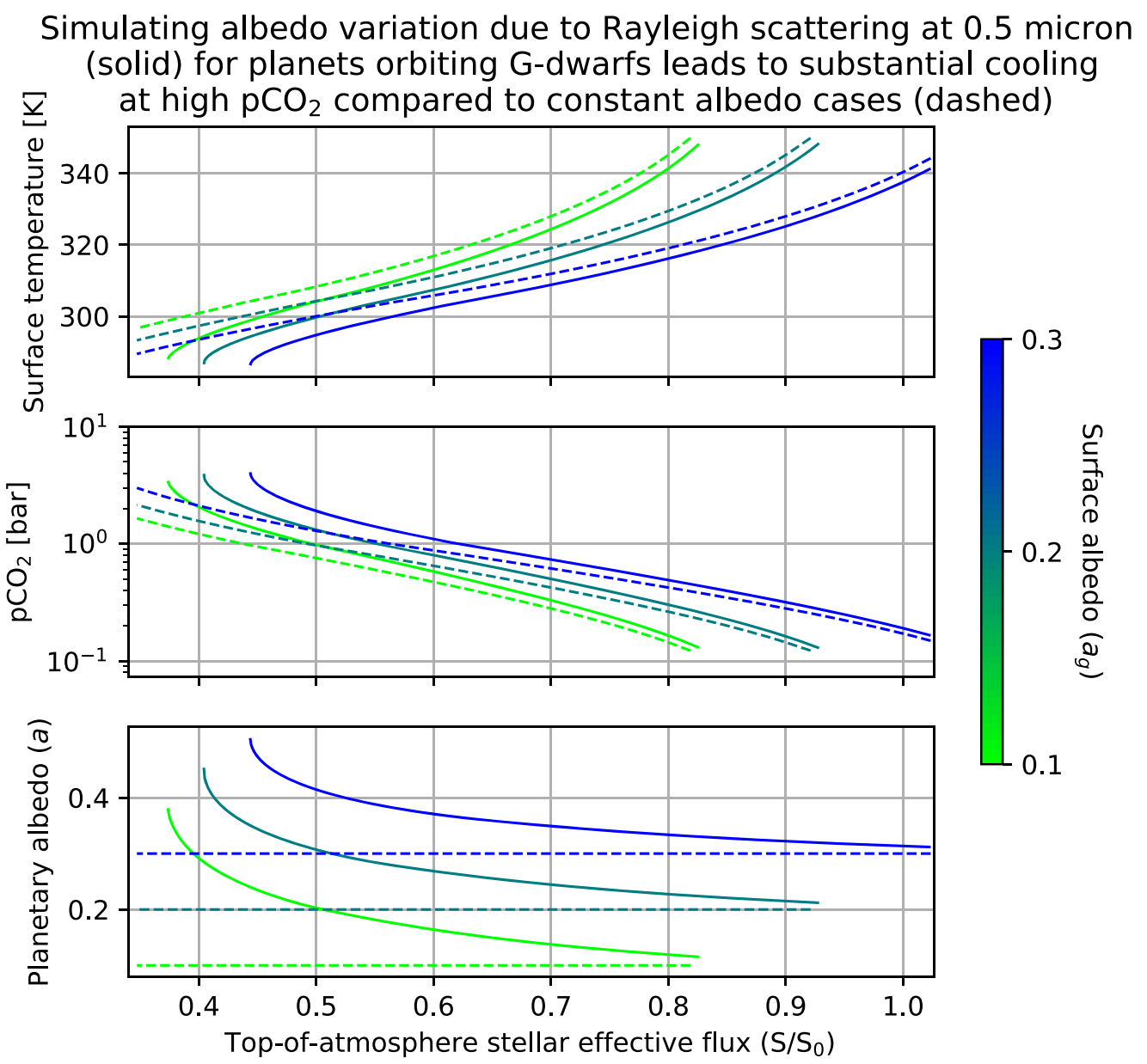

Figure 6. Comparison of temperature, $\mathrm{pCO}_{2}$, and planetary albedo vs. instellation in MAC simulations with (solid curves) and without (dashed curves) planetary albedo that varies as a function of $\mathrm{pCO}_{2}$ and surface albedo. The top plot shows relative instellation - the ratio of top-of-atmosphere instellation to modern-day Earth instellation $\left(S / S_{0}\right.$, where $\left.S_{0}=1368 \mathrm{~W} \mathrm{~m}^{-2}\right)$-vs. temperature $(T)$. The middle plot shows relative instellation vs. $\mathrm{pCO}_{2}$. The bottom plot shows relative instellation vs. planetary albedo. The bluest curves in each plot have a surface albedo $a_{g}=0.3$, the blue-green curves have $a_{g}=0.2$, and the green curves have $a_{g}=0.1$. The thermodynamic coefficient $\Lambda=\Lambda_{0} / 5$ in these simulations so that climates would reach high enough $\mathrm{pCO}_{2}$ for Rayleigh scattering to become important. Other parameters take their default values.

Figure 6). This cooling effect moves the effective outer edge of the $\mathrm{HZ}$ closer to the star.

\subsection{Impact of an Energetic Limit on Precipitation}

In steady state, precipitation is in equilibrium with evaporation, which is ultimately driven by instellation. This implies a maximum planetary precipitation rate constrained by planetary instellation (Equation (43)), a prediction that is borne out by both 1D (Pierrehumbert 2002) and 3D (O'Gorman \& Schneider 2008; le Hir et al. 2009) climate models.

\subsubsection{Energetic Limit without Land Fraction Dependence}

The impact of the energetic limit on precipitation is illustrated in Figure 5. Reducing $\Lambda$ (the thermodynamic coefficient in Equation (20)) reduces $C_{\text {eq }}$ for a given $\mathrm{pCO}_{2}$, which increases the temperature, $\mathrm{pCO}_{2}$, and precipitation necessary to achieve a runoff high enough to balance outgassing. Simulations with $\Lambda=\Lambda_{0} / 5$ and above (where $\Lambda_{0}$ is the default value for $\Lambda$ listed in Table 1) have temperature slopes identical to those of their precipitation curves because precipitation is linear with respect to temperature. However, for the simulations with $\Lambda_{0} / 8$ and $\Lambda_{0} / 10$, temperature eventually becomes decoupled from precipitation and begins to increase instead of decrease as instellation falls. This is because the temperatures in these simulations are forced so high by the restriction of $C_{\mathrm{eq}}$ that they each achieve the maximum precipitation at a given instellation somewhere in the HZ. For simulations that reach their maximum precipitation rate at some instellation, precipitation then follows $p_{\lim }$ and decreases linearly with instellation beyond that (note that the red and red-orange precipitation curves converge onto the same line in the bottom panel of Figure 5, as they both follow Equation (43)). Because precipitation (and therefore runoff) decreases faster with instellation when governed by Equation (43), this forces $\mathrm{pCO}_{2}$ to grow at a higher rate with decreasing instellation in order to increase $C_{\mathrm{eq}}$ and allow the solute to accumulate to high enough concentrations to maintain a weathering flux balanced with outgassing despite reduced runoff (see the bottom panel of Figure 1 to see the relationship between $\mathrm{pCO}_{2}$, runoff, and weathering flux for a given set of parameters). This increased $\mathrm{CO}_{2}$ accumulation per unit instellation reduction is what leads to the counterintuitive temperature increase at low instellations in the red and redorange curves in the top panel of Figure 5. 


\section{Simulations using $p_{\text {lim, land }}$ (Eq. 45; solid) for the energetic limit on precipitation are warmer than those using $p_{\text {lim }}$ (Eq. 43; dashed) and behave differently as a function of $\gamma$}
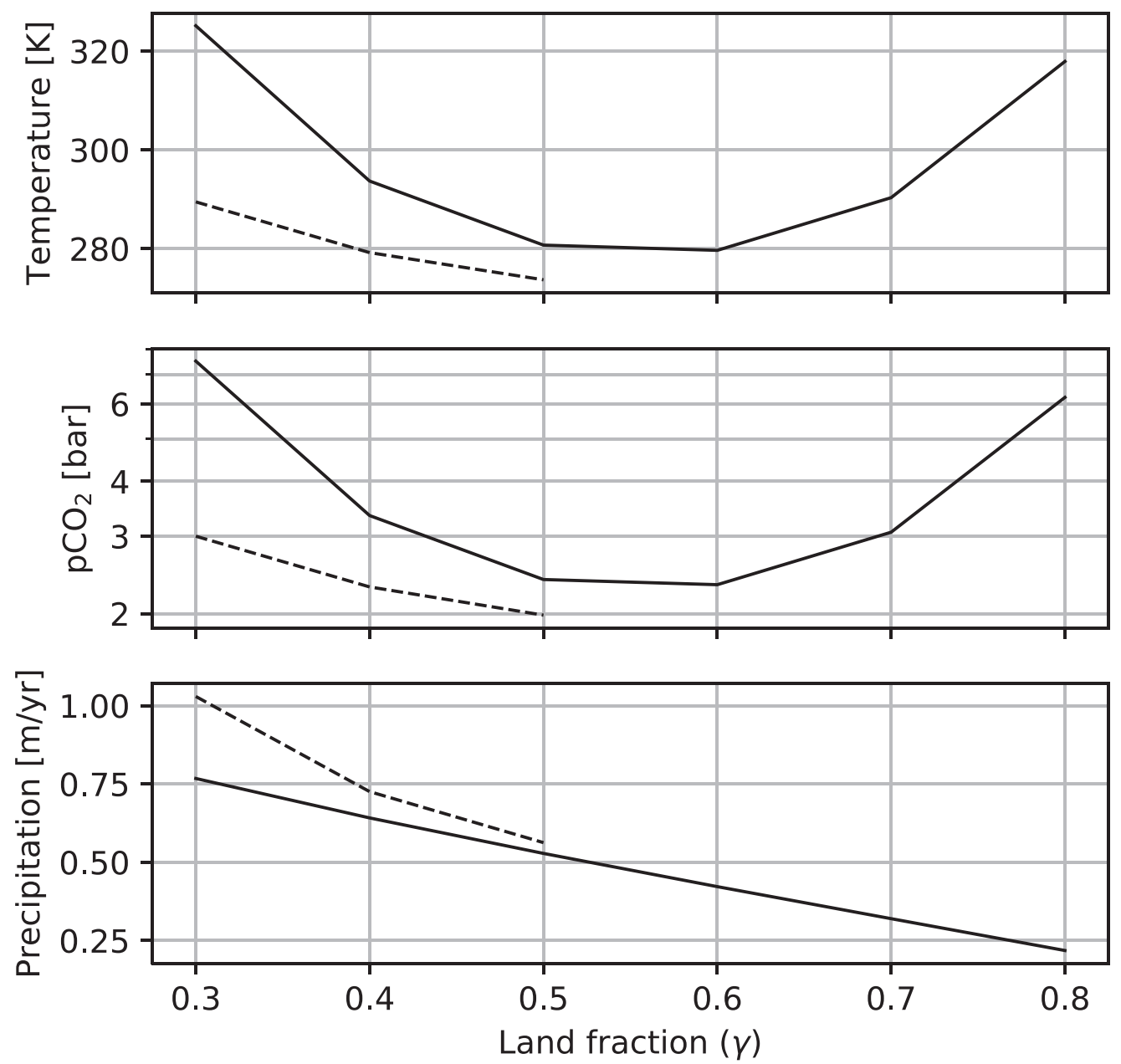

Figure 7. Comparison of temperature, $\mathrm{pCO}_{2}$, and precipitation at the classical outer edge of Earth's $\mathrm{HZ}\left(S / S_{0}=0.346\right.$ (Kopparapu et al. 2013)) as a function of land fraction $(\gamma)$ for planets with an energetic limit on precipitation that either scales with ocean surface fraction (solid curves; Equation (45)) or is independent of ocean surface fraction (dashed curves; Equation (43)). The top panel shows $\gamma$ vs. temperature (T), the middle panel shows $\gamma$ vs. pCO ${ }_{2}$, and the bottom panel shows $\gamma$ vs. precipitation $(p)$. The simulations have thermodynamic coefficient $\Lambda=\Lambda_{0} / 5$, but other parameters take their default values.

\subsubsection{Energetic Limit with Land Fraction Dependence}

We also ran a set of simulations with $p_{\text {lim,land }}$ (Equation (45)) instead of $p_{\text {lim }}$ (Equation (43)) to examine the impact of scaling the energetic limit on global precipitation by surface ocean fraction $(1-\gamma)$. This leads to several changes in the weathering behavior of planets in the energetically limited regime as a function of land fraction $\gamma$. This is demonstrated in Figure 7, which compares the temperature, $\mathrm{pCO}_{2}$, and precipitation for simulations with thermodynamic coefficient $\Lambda=\Lambda_{0} / 5$ at $S / S_{0}=0.346$ (the classical outer edge of the $\mathrm{HZ}$ for an Earth-mass planet around a G2 star according to Kopparapu et al. 2013) with and without the new scaling law for the energetic limit.

The first point to note is that scaling the energetic limit with ocean fraction lowers the temperature at which a planet's precipitation becomes energetically limited for a given instellation, as a reduced fraction of the instellation is available to drive precipitation. For the simulations that use $p_{\text {lim }}$, a thermodynamic coefficient of $\Lambda_{0} / 5$ (with other parameters set to default values) leads to a surface temperature that is not high enough to enter the energetically limited regime at any instellation (see Figure 5 and the dashed curves in Figure 7) regardless of land fraction; in contrast, simulations that use $p_{\text {lim,land }}$ instead of $p_{\text {lim }}$ enter the energetically limited regime for all $\gamma$ when $\Lambda=\Lambda_{0} / 5$ (see solid curves in Figure 7). Another difference between these sets of simulations is that energetically limited simulations that use $p_{\text {lim,land }}$ tend to display warmer temperatures and higher $\mathrm{pCO}_{2}$ than those using $p_{\text {lim }}$ (see the top and middle panels of Figure 7). This is because simulations with $p_{\text {lim,land }}$ are restricted to a lower maximum precipitation rate (see the bottom panel in Figure 7). A lower precipitation rate implies a lower rate of runoff in our models, which forces $\mathrm{pCO}_{2}$ to build to higher levels in order to increase 
the thermodynamic limit $C_{\mathrm{eq}}$ enough for the low runoff to deliver sufficient cations to the ocean to balance outgassing of $\mathrm{CO}_{2}$.

Finally, there is a qualitative difference in the dependence of planetary climate and weathering behavior on land fraction $(\gamma)$ for energetically limited planets with $p_{\text {lim,land }}$. In our simulations, under conditions not limited by energy (and under energetically limited conditions governed by $p_{\text {lim }}$ instead of $\left.p_{\text {lim,land }}\right)$, increased land fraction leads to cooling (e.g., Figure 2 or the leftmost column of Figure 3). This is because a larger land area allows a given outgassing rate to be balanced with a smaller weathering flux per unit land surface, necessitating lower precipitation rates and temperatures. In contrast, energetically limited simulations with $p_{\text {lim,land }}$ may warm or cool with increased $\gamma$ (see the top panel of Figure 7). When cooling dominates, it is because of the effect just discussed. When warming occurs with increased land fraction, it is because $p_{\text {lim,land }}$ (and therefore runoff) becomes very small at high $\gamma$ as the ocean surface fraction becomes small, which forces $\mathrm{pCO}_{2}$ to increase greatly so that $C_{\text {eq }}$ becomes large enough that the tiny rates of runoff can still deliver enough cations to the ocean to balance outgassing.

\section{Discussion and Conclusions}

\subsection{Discussion}

\subsubsection{The Influence of Tectonic Mode on Silicate Weathering}

In this study, we did not address the tectonic regime of the planets we modeled, but weathering is probably strongly impacted by tectonic mode. Planets with plate tectonics have a robust mechanism for removing weathered material and delivering fresh weatherable material to the planetary surface, potentially improving their ability to avoid a "supply-limited" weathering regime where the negative feedback between climate and weathering is lost due to depletion of weatherable minerals (West et al. 2005; Foley 2015). Simulations of plate tectonic planets display efficient $\mathrm{CO}_{2}$ degassing under most conditions (Noack et al. 2014). Simulations of stagnant lid planets suggest that they may also be able to avoid the supply limit over geologic timescales (Foley \& Smye 2018; Foley 2019) and display efficient degassing and volcanism under a range of conditions, though their volcanic activity may become limited at high core-mass fraction or high planetary mass (Noack et al. 2014, 2017; Dorn et al. 2018). Between these endmembers, there are intermediate states like the "episodic" mode, with long periods of stagnant lid behavior punctuated by periods of relatively rapid resurfacing and outgassing (Lenardic et al. 2016).

The MAC formulation suggests some natural ways to explicitly incorporate tectonic mode into simulations of planetary weathering behavior. Through its impact on the Damköhler coefficient $\left(D_{w}\right)$, the average soil age of material being weathered in our simulations $\left(t_{s}\right)$ has a powerful impact on the weathering behavior of rocky planets. Young soils lead to large values of $D_{w}$, allowing for a robust weathering feedback. Ancient soils that are depleted in reactive minerals can enter a supply-limited weathering regime. We would expect that planets with more rapid resurfacing will, on average, have younger soils and therefore greater values of $D_{w}$. They should also have higher volcanic outgassing rates. Younger soils lead to more efficient weathering and therefore lower equilibrium $\mathrm{pCO}_{2}$ and temperatures. Higher outgassing rates lead to higher equilibrium $\mathrm{pCO}_{2}$ and temperature. Because greater volcanic activity may lead to both faster resurfacing and higher rates of outgassing, the effects tend to offset one another, and it is difficult to predict what the net impact on planetary weathering behavior would be.

Tectonic mode should also influence the lithology of the material being weathered on a given planet. For example, the relative abundances of granitic and basaltic weatherable material may be determined by the relative rates of continental generation versus production of flood basalts, which will in turn be controlled by the primary mode of resurfacing on a planet. Ibarra et al. (2016) showed that granitic and basaltic river catchments on Earth display divergent weathering behavior: rivers draining basaltic catchments tend to have higher values of $C_{\mathrm{eq}}$ and cation concentration. Winnick \& Maher (2018) argued that the weathering of basaltic minerals should display greater sensitivity to $\mathrm{pCO}_{2}$ because basalts have a higher proportion of divalent cations, which leads to a larger value for the thermodynamic $\mathrm{pCO}_{2}$ sensitivity ( $n$; see Equations (19) and (20)). Those results imply that planetary weathering dominated by granites versus basalts could lead to quite different equilibrium climate states for planets with otherwise similar properties.

We also note the importance of mountain-building processes for the silicate weathering feedback. Much of the chemical weathering on Earth takes place in mountainous regions where fresh minerals are exposed to Earth's surface and soil is produced and eroded at high rates (Larsen et al. 2014a, 2014b). Because orogeny is heterogeneous in space and time on Earth, the build-up and grind-down of mountains, as well as the climatic conditions at the locations on Earth where these processes take place, may have a profound impact on the carbon cycle and global climate of a planet through time. Periods of active mountain-building (particularly in areas with appreciable runoff) may lead to global cooling by increasing the fraction of fresh minerals $\left(f_{w}\right.$; equivalent to reducing soil age) in the region being weathered, whereas periods of slow uplift may allow $\mathrm{CO}_{2}$ to accumulate to high levels in the atmosphere (Raymo \& Ruddiman 1992; Jagoutz et al. 2016; Kump 2018; Macdonald et al. 2019)

\subsubsection{Relation to Previous Rocky Exoplanet Weathering Results}

To our knowledge, all previous rocky exoplanet weathering studies have used some variant of the WHAK formulation to represent weathering. Simulations with the MAC formulation display some important differences in behavior compared to those with the WHAK formulation. So, if we assume that the MAC formulation is an accurate representation of planetary silicate weathering, some conclusions of these previous studies should be revisited. We survey a few points of interest below.

As we noted in Section 4, Abbot et al. (2012) and Foley (2015) found that planetary weathering behavior is relatively insensitive to land fraction with the WHAK model. We found the opposite with the MAC model: land fraction strongly impacts the planetary temperature, particularly at low land fraction. This suggests that understanding the processes that control oceanic volume (e.g., Kasting \& Holm 1992; Cowan \& Abbot 2014; Schaefer \& Sasselov 2015; Komacek \& Abbot 2016) and the development of continents (e.g., Rosing et al. 2006; Höning et al. 2014, 2019; Höning \& Spohn 2016) on exoplanets is extremely important for predicting the frequency of occurrence of truly habitable planets. 
Snowball limit cycling is a hypothetical phenomenon on terrestrial planets where silicate weathering draws down $\mathrm{CO}_{2}$ faster than outgassing supplies it, even at temperatures cold enough to freeze a planet, plunging a planet into the snowball state. In the snowball state, silicate weathering is expected to slow down or cease, allowing $\mathrm{CO}_{2}$ to outgas and build up until a planet has warmed enough to deglaciate, at which point rapid silicate weathering freezes it again (Tajika 2007; Menou 2015; Abbot 2016; Batalha et al. 2016; Haqq-Misra et al. 2016; Paradise \& Menou 2017; Ramirez 2017; although $\mathrm{CO}_{2}$ condensation in the extreme cold of the snowballs or continued weathering at the seafloor during the snowball may make deglaciation difficult under some conditions; Turbet et al. 2017; Kadoya \& Tajika 2019). In simulations with the WHAK formulation, limit cycling is driven by the kinetic dependence of weathering on $\mathrm{pCO}_{2}(\beta)$ increasing weathering rates at low instellations where $\mathrm{pCO}_{2}$ is very high. In the MAC formulation, kinetic $\mathrm{pCO}_{2}$ dependence has essentially no impact on planetary weathering rates (see the middle column of Figure 3), but the thermodynamic dependence of $C_{\text {eq }}$ on $\mathrm{pCO}_{2}$ leads to a similar result. Many parameter configurations lead to planets with equilibrium temperatures below freezing at instellations within the HZ, so planets with these parameter sets at low enough instellations would either be locked into a permanent snowball state or go through limit cycling. The fact that factors like hydrology and soil age and lithology impact the effective outer edge of the HZ so strongly (see the top row in Figure 4) implies that a planet's susceptibility to limit cycling (and therefore its habitability) is determined by a complex interplay of factors that are not easily constrained a priori. Arguing that a given planet (e.g., Mars; Batalha et al. 2016) experienced limit cycling thus requires making many implicit and potentially unfounded assumptions about the properties that controlled its weathering (see Ramirez 2017 and Hayworth et al. 2020) for further discussion of the early Martian limit cycling hypothesis).

Kite et al. (2011) use WHAK formulation of weathering to argue that there may be conditions where silicate weathering acts as a destabilizing feedback on the climates of rocky tidally locked planets. The "enhanced substellar weathering instability" (ESWI) is proposed to take place on tidally locked planets with relatively thin atmospheres composed largely of $\mathrm{CO}_{2}$. Because heat transport in thin atmospheres decreases with reductions in density, a decrease in atmospheric mass can lead to net warming at the substellar point on a tidally locked planet despite the reduction in greenhouse effect, due to the decreased ability of the atmosphere to export solar energy away from the substellar point. Because of the exponential dependence of weathering rates on temperature in the WHAK formulation, the vast majority of weathering on a tidally locked planet occurs near the substellar point, so an increase in temperature at the substellar point through a reduction in atmospheric pressure can enhance global weathering significantly. The enhanced weathering would in turn draw down more $\mathrm{CO}_{2}$, again reducing atmospheric pressure, warming the substellar point, and enhancing weathering. This is a positive feedback leading to atmospheric collapse, because weathering begets more weathering, and the reverse scenario of runaway $\mathrm{CO}_{2}$ accumulation also occurs in the case of an initial increase in atmospheric mass (or an initial cooling). However, the ESWI depends intimately on the strength of the temperature dependence of the silicate weathering feedback. The nonexponential temperature dependence in the MAC formulation might lead to global weathering that is less concentrated at the substellar point, implying that tidally locked planets with thin $\mathrm{CO}_{2}$-dominated atmospheres may be less vulnerable to the ESWI than suggested by Kite et al. (2011).

\subsubsection{Implications for Early Earth Climate Evolution}

During Earth's Archean eon (4-2.5 Ga), the Sun was likely 20\%-30\% fainter than today (e.g., Sagan \& Mullen 1972; Bahcall et al. 2001). Sparse proxy estimates from this period seem to imply a temperate world with ocean temperatures $<40^{\circ} \mathrm{C}$ and occasional partial glaciations (e.g., Hren et al. 2009; Blake et al. 2010; Ojakangas et al. 2014; de Wit \& Furnes 2016; Catling \& Zahnle 2020), suggesting the climate was effectively buffered against reduced luminosity. The question of how Earth maintained habitability despite this large change in luminosity is sometimes referred to as the "faint young Sun problem," a particularly famous component of the more general problem of constraining the evolution of the climate during Earth's deep prehistory. As we noted in the introduction, the silicate weathering feedback is often invoked as a source of the climatic buffering in Earth's past, and this interpretation is consistent with carbon cycle models of the Archean period that use WHAK-style kinetic representations of continental and seafloor weathering to estimate Archean Earth's equilibrium climate state (e.g., Charnay et al. 2017). In fact, the proxy record of the ancient climate has been combined with the WHAK-based inverse geologic carbon cycle modeling in attempts to simultaneously provide tighter constraints on Earth's temperature- $\mathrm{CO}_{2}$ history, key globally averaged parameters like $T_{e}$ and $\beta$ in the WHAK formulation, and the relative importance of processes like seafloor weathering and reverse weathering compared to continental weathering in the past (Krissansen-Totton \& Catling 2017, 2020; Krissansen-Totton et al. 2018). These examples illustrate how the WHAK framework, as a central component of most models of the carbon cycle of ancient Earth, contributes to current understanding of the Archean Earth system and the faint young Sun problem.

As shown in Section 4.1, the MAC and WHAK formulations of weathering lead to different atmospheric responses to changes in boundary conditions. MAC is much more sensitive to changes in land fraction $(\gamma)$ and volcanic outgassing $(v)$, displaying a $30-40 \mathrm{~K}$ temperature increase in response to a factor of 2 reduction in $\gamma / v$, compared to a $\sim 5 \mathrm{~K}$ increase for WHAK (see Figure 2 and the leftmost column of Figure 3, noting that reductions in $v$ are equivalent to proportional increases in $\gamma$ ). On the other hand, depending on parameter choices like $n$ and $\beta$, either formulation of weathering can be more sensitive to changes in luminosity. On Earth, all of these boundary conditions have changed substantially since the Archean, with a general secular decrease in outgassing by as much as a factor of 10 (Avice et al. 2017) and an increase in dry land by a factor of 10 or more (Flament et al. 2008; Johnson \& Wing 2020) accompanying the increase in solar luminosity already mentioned. This suggests an increase in $\gamma / v$ by perhaps a factor of $\sim 100$ (!) since the Archean, which is enormous compared to the factor of 2 reduction that causes $30-40 \mathrm{~K}$ of warming in our default MAC model. Of course, other parameters in the MAC model have certainly changed since the Archean-we specifically discuss these boundary conditions because the magnitude of change over time and the divergence in response by WHAK and MAC help illustrate the 
point that replacing the WHAK formulation in carbon cycle models with a MAC-style parameterization could lead to substantially different conclusions about the evolution of the Earth system since the Archean.

Because the change in luminosity sensitivity between the two formulations can go in either direction, we will also ignore that potential difference between WHAK and MAC, and focus on sensitivity to land fraction and outgassing. Because MAC weathering requires larger increases in temperature than WHAK to maintain equilibrium in response to reduced $\gamma / v$, and because $\gamma / \nu$ may have been a factor of 100 smaller during the Archean, replacing the WHAK model with the MAC formulation would lead to a much hotter inferred Archean climate, other things being equal. However, as noted in the beginning of this section, the (very limited) climate proxy estimates from the Archean suggest a world with a temperate climate not much warmer than our own. Assuming those estimates are trustworthy, the behavior of the MAC model may make it necessary to invoke other changes to the carbon cycle, e.g., a greater seafloor weathering flux, to generate enough weathering to balance outgassing at temperatures consistent with the mild climate implied by proxies. Other differences, particularly the lack of vascular land plants, may imply further weakening of Archean continental weathering (e.g., Rafiei \& Kennedy 2019), though the influence of plants is still poorly understood. These suggestions can be tested quantitatively. The implications of the MAC formulation for our interpretation of the proxy record of the evolution of the Earth system should be addressed rigorously with a statistical methodology that accounts for the huge uncertainties in most parameters when modeling the ancient carbon cycle (see the approach taken in Krissansen-Totton \& Catling 2017, 2020; Krissansen-Totton et al. 2018).

\subsubsection{Observability of the Silicate Weathering Feedback}

Bean et al. (2017) and Checlair et al. (2019a) suggest the use of "statistical comparative planetology" to detect the operation of the silicate weathering feedback on rocky planets in the HZ. Specifically, Bean et al. (2017) and Checlair et al. (2019a) propose using a relatively large number of low-cost, lowprecision $\mathrm{CO}_{2}$-abundance measurements of rocky planets throughout the $\mathrm{HZ}$ with a future telescope to show that $\mathrm{pCO}_{2}$ tends to decrease with increases in instellation, which would provide evidence for a stabilizing feedback on planetary climate. With the assumption that the silicate weathering feedback would adjust $\mathrm{pCO}_{2}$ to hold planetary temperatures at $280 \mathrm{~K}$, and with very optimistic assumptions for observational noise, Checlair et al. (2019a) conclude that $\sim 10$ planets with functioning silicate weathering feedbacks in the $\mathrm{HZ}$ would need to have their $\mathrm{CO}_{2}$ partial pressures characterized to give an $80 \%$ chance of detecting a trend in $\mathrm{pCO}_{2}$ versus instellation. Under pessimistic instrumental noise assumptions, Checlair et al. (2019a) calculate that the number of necessary characterizations increases to $\sim 50$. These numbers bracket the range of estimated yields for "exo-Earth" planets from proposed next-generation telescopes HabEx (Gaudi et al. 2020) and LUVOIR (The LUVOIR Team 2019). However, it is worth noting that recent studies suggest that the occurrence rate of Earth-like planets may be up to an order of magnitude lower than the numbers used to calculate those estimates (Pascucci et al. 2019; Neil \& Rogers 2020), which would lead to a proportional reduction in yield.
Our study suggests a larger number of observations may be required to establish a trend of $\mathrm{pCO}_{2}$ versus instellation and confirm the operation of the silicate weathering feedback, due to the huge variations in $\mathrm{pCO}_{2}$ and temperature at a given instellation for planets with different outgassing rates or combinations of hydrological, thermodynamic, and surface mineralogical parameters. As an example, see the middle panel in Figure 5: at $S / S_{0} \sim 0.9$, equilibrium $\mathrm{pCO}_{2}$ varies by four orders of magnitude between the simulations with $\Lambda=\Lambda_{0} / 10$ and $10 \Lambda_{0}$. Both of those values of $\Lambda$ are within the range expected from the diversity of silicate mineral assemblage lithologies (Winnick \& Maher 2018). Varying other parameters produces a similar spread in $\mathrm{pCO}_{2}$. Effectively, the potential diversity in weathering behavior among terrestrial planets adds a form of intrinsic "noise" to the statistical estimates in Checlair et al. (2019a). To add to the complication, variations in seafloor weathering or reverse weathering (discussed below in Section 5.1.6) could also add to the variety of climates encountered in the HZ.

The diversity in equilibrium climates permitted by variations in hydrological, thermodynamic, and surface parameters, coupled with the potentially low occurrence rate of Earth-like planets noted above, suggests that the method outlined in Bean et al. (2017) and Checlair et al. (2019a) may not be adequate to detect the silicate weathering feedback with upcoming telescope missions. Because of this, we suggest that a complementary method first suggested in Turbet (2020) to identify the breakdown of the silicate weathering feedback at the inner edge of the $\mathrm{HZ}$ may be able to successfully demonstrate the existence of the feedback with a smaller number of observations. We do not statistically quantify the number of characterizations necessary to identify the operation of the silicate weathering feedback with this method, as that is beyond the scope of the current study. The method from Turbet (2020) that we will discuss makes use of the potential for a large discontinuity in $\mathrm{CO}_{2}$ concentration between planets on opposite sides of the inner edge of the HZ, defined here as the instellation above which a planet enters the runaway greenhouse state and loses its water to space (Kasting et al. 1993). There is not a unique instellation where the runaway greenhouse occurs, as the critical irradiation level depends on planetary rotation rate (Yang et al. 2014), planetary radius (Yang et al. 2019), surface gravity (Yang et al. 2019), stellar temperature (Kopparapu et al. 2013), background gas partial pressure (Ramirez 2020), surface water distribution (Kodama et al. 2018, 2019), and cloud properties (Leconte et al. 2013), but for the purposes of this discussion, we will use a conservative estimate for an Earth-mass, rapidly rotating planet around a Sun-like star given in Kopparapu et al. (2013). In that study, the critical instellation for a planet with those properties is found to be $S_{\text {eff }}=1.05$ (where $S_{\text {eff }}=S / S_{\text {earth }}$ is the ratio of flux received by the planet to the flux received by modern Earth).

For planets just beyond the inner edge (e.g., planets just within the $\mathrm{HZ}$ ) with a functional silicate weathering feedback, $\mathrm{CO}_{2}$ will be drawn down to low levels due to the high instellation. To give a conservative maximum estimate of $\mathrm{pCO}_{2}$ at the inner edge of the HZ, we use Equation (31) to find the $\mathrm{pCO}_{2}$ necessary to equilibrate a planet with an albedo of 0.3 and $S_{\text {eff }}=1.05$ at a surface temperature of $340 \mathrm{~K}$. This yields a $\mathrm{pCO}_{2}$ of 0.05 bar. In actuality, the $\mathrm{pCO}_{2}$ could be considerably lower, depending on the various parameters that control the 


\section{The large contrast in $\mathrm{pCO}_{2}$ and albedo between planets on opposite sides of the inner edge of the $\mathrm{HZ}$ may provide observable evidence of the silicate weathering feedback}

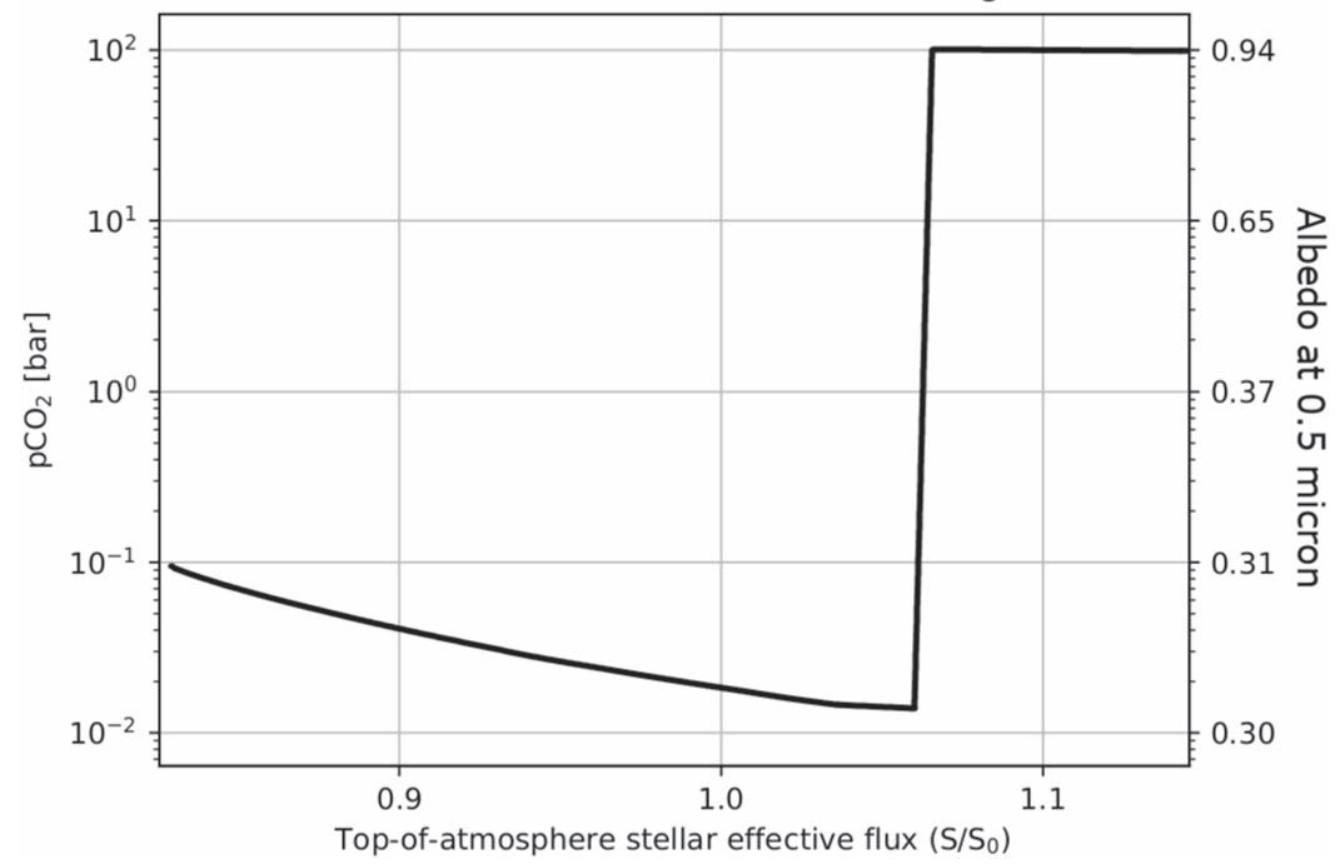

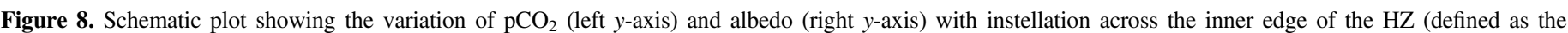

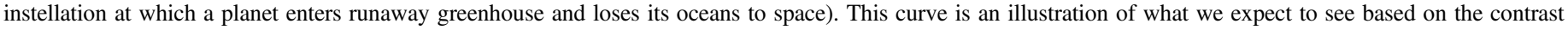

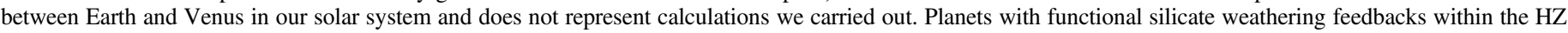

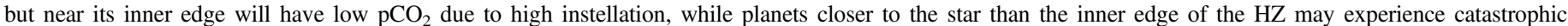

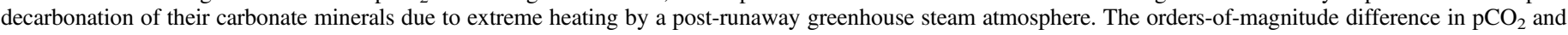

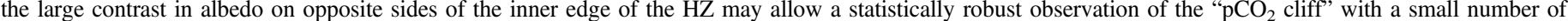

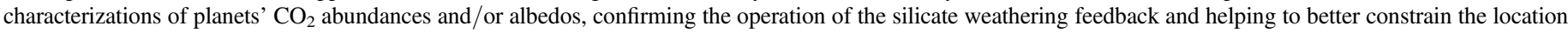

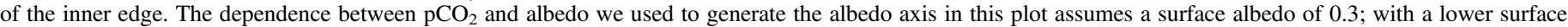
albedo, the contrast in albedos across the inner edge of the $\mathrm{HZ}$ would be even larger.

weathering rate, but with a higher $\mathrm{pCO}_{2}$, a planet would be hot enough to enter a moist greenhouse regime and lose its water to space efficiently (Kasting et al. 1988).

In contrast, Earth-like planets within the inner edge of the $\mathrm{HZ}$ that undergo a runaway greenhouse and lose their oceans to space may end up in a "Venus-like" state with $\sim 100$ bars of $\mathrm{CO}_{2}$ in their atmosphere due to the catastrophic decarbonation of their mineral inventories under extreme temperatures (though this prediction depends on the magnitude of carbon delivery to a planet during its accretion phase). Even in the absence of catastrophic decarbonation, if a planet loses its water due to runaway greenhouse but still has even a little bit of $\mathrm{CO}_{2}$ outgassing (and an Earth-like inventory of carbon), a Venus-like $\mathrm{CO}_{2}$-rich atmosphere will accumulate, as a fully dry planet cannot maintain a silicate weathering feedback. This suggests that there could be a two to three orders-of-magnitude discontinuity in $\mathrm{pCO}_{2}$ for Earth-like planets straddling opposite sides of the inner edge of the HZ, assuming an operative silicate weathering feedback within the HZ.

If present, the multiple-orders-of-magnitude change in $\mathrm{pCO}_{2}$ across the inner edge of the $\mathrm{HZ}$ (see Figure 8 for a schematic illustration of this concept) may be observable with nextgeneration telescopes like the Origins Space Telescope (OST), HabEx, or LUVOIR without the need for a large number of atmospheric characterizations. However, it may be difficult to demonstrate that a planet has $\mathrm{CO}_{2}$ partial pressures greater than a bar or two via infrared emission, because $\mathrm{CO}_{2}$ becomes optically thick throughout the IR region at high column abundances; for example, remote observations of the nightside of Venus look similar to the dayside of Mars in the IR despite a factor of $\sim 16,000$ difference in $\mathrm{CO}_{2}$ partial pressure, because IR only escapes from the top bar of Venus's atmosphere (see Figure 3 in Pierrehumbert 2011).

In the absence of direct measurements of atmospheric $\mathrm{pCO}_{2}$ at the surface of Venus-like planets, we propose that the contrast in albedo across the inner edge of the $\mathrm{HZ}$ at visible wavelengths due to Rayleigh scattering by $\mathrm{CO}_{2}$ should be substantial and likely observable: low- $\mathrm{pCO}_{2}$ planets near the inner edge of the $\mathrm{HZ}$ would have albedos approximately equal to that of their surface + clouds, suggesting a maximum of around 0.3 (based on Earth's albedo in the visible; Tinetti et al. 2006), whereas Venus-like planets would have visiblewavelength albedos $>0.9$ due to efficient Rayleigh scattering by $\sim 100$ bar of $\mathrm{CO}_{2}$, even in the absence of shiny sulfuric acid clouds like those hosted by Venus (calculated at $0.5 \mu \mathrm{m}$ with Equation (30); see also Table 5.4 in Pierrehumbert 2010). Note that we are specifically discussing albedo in the visible region of the spectrum: albedo integrated across the spectrum would include near-infrared absorption effects, which would reduce the albedo contrast across the inner edge of the $\mathrm{HZ}$ and introduce a dependence on stellar type because lowertemperature stars emit preferentially at longer wavelengths. 
By restricting the discussion to visible-wavelength albedo, we avoid those complications.

In summary, observations of large differences in $\mathrm{pCO}_{2}$ and/ or visible-wavelength albedo between planets on opposite sides of the inner edge of the $\mathrm{HZ}$ may allow for a demonstration that the silicate weathering feedback stabilizes planetary climate within the $\mathrm{HZ}$ and fails to stabilize climate outside of the $\mathrm{HZ}$. In particular, because of the abrupt and large change in $\mathrm{pCO}_{2}$ and albedo across the inner edge of the HZ, the existence of the silicate weathering feedback may be demonstrable with a smaller number of observations than a method that depends on the potentially noisy trend of $\mathrm{pCO}_{2}$ versus irradiation due to silicate weathering within the $\mathrm{HZ}$.

\subsubsection{Relating Planetary Characteristics to Weathering Controls}

The potential diversity in climate outcomes we discuss above might partially be from our ignorance of the constraints on parameters controlling weathering in the MAC formulation. A better understanding of the likely distributions of important variables like soil thickness and age, particularly as functions of bulk parameters like planetary mass, might serve to narrow the range of climate states we expect Earth-like planets with MACstyle weathering to exhibit. Without monumental advances in observational techniques (e.g., Turyshev et al. 2020), direct observation of these parameters on exoplanets is not feasible. Even on Earth, where observations are many orders of magnitude more dense and sensitive than they ever could be for an exoplanet, quantitative understanding of the factors controlling weathering is in its early stages. However, progress is still possible through a combination of theory, modeling, generalization from Earth observations where appropriate, and, eventually, "statistical comparative planetology" of the sort described by Bean et al. (2017) and Checlair et al. (2019a).

Theory and modeling can be used to deepen understanding of the relationships between the parameters controlling weathering. In this study, we ignored a variety of potentially important feedbacks between variables in the MAC model. For example, a greater erosion rate decreases soil thickness $(\sim L)$, increasing the production rate of soil (Heimsath et al. 2000), leading to a higher fraction of fresh, weatherable minerals in the soil column $\left(f_{w}\right)$. The reduction in soil thickness tends to reduce $D_{w}$ and solute concentration, while the increase in $f_{w}$ has the opposite effect, and these opposing responses produce a "humped" functional dependence of weathering on erosion, where weathering rates increase with increasing erosion up to a point, beyond which weathering decreases (see Figure S5 in the supplement to Maher \& Chamberlain 2014 for calculations showing this effect). In turn, erosion rates depend on precipitation (Perron 2017), topographic relief (Montgomery $\&$ Brandon 2002), and vegetation cover (Collins et al. 2004), among other things. Accounting for the feedback between precipitation and erosion would lead to a stronger coupling between weathering and temperature under circumstances where increased erosion increases weathering rates, as seems to be the case on Earth, where erosion and weathering rates are positively correlated (e.g., Gaillardet et al. 1999). This may make extremely warm climates less likely to occur than a random sampling of parameter combinations in the MAC model would suggest, placing tighter constraints on likely $\mathrm{pCO}_{2}$ values for planets with operative weathering feedbacks. Similarly, because tectonic uplift generates topographic relief which accelerates erosion, we would expect planets with greater globally averaged uplift rates and/or more extreme topography to tend to be cooler, all else being equal. Topography and uplift are determined by the tectonic state of a planet, which is probably strongly tied to variables like the mass and age (thermal history) of a planetary system. This suggests that continued development of theory surrounding the tectonics of Earth-like planets (see, e.g., O’Neill 2012 for a discussion) could point toward observable correlations between HZ climate and bulk parameters like planetary mass or age. Depending on the expected size of the effects, such trends may be observable with OST/HabEx/LUVOIR-generation telescopes, a variant of the weathering feedback detection method discussed in Checlair et al. (2019a).

\subsubsection{Limitations of This Study}

An important limitation of this study is the exclusion of seafloor weathering. Oceanic crust alteration is an important sink of $\mathrm{CO}_{2}$ on Earth, and it is possible that this form of weathering is temperature or $\mathrm{pCO}_{2}$ dependent, which implies that it may act as a negative feedback on Earth's climate in addition to continental silicate weathering (e.g., Brady \& Gislason 1997; Coogan \& Gillis 2013; Coogan \& Dosso 2015). For example, Krissansen-Totton et al. (2018) found that the inclusion of temperature- and $\mathrm{pH}$-dependent seafloor weathering significantly moderated climate variations in a geological carbon cycle model of Earth's deep past. If seafloor weathering really does act as a negative feedback on climate, then the sensitivity of climate to land fraction, outgassing, and continental surface properties that we found with the MAC formulation of continental weathering should be less extreme. Our results are intended to highlight the different effects of MAC versus WHAK continental weathering and correspond to an extreme limit in which seafloor weathering does not act as a stabilizing or destabilizing feedback. Habitability calculations would be improved by an accurate model of seafloor weathering, but this presents a considerable challenge, as there is no clear consensus as to the nature of the seafloor weathering feedback (e.g., Caldeira 1995), so we did not attempt to model this process. We do, however, suggest that the thermodynamic limit on solute concentration may also be relevant for seafloor weathering: ocean bottom water percolating through hydrothermal systems may reach a maximum concentration of solute analogous to $C_{\mathrm{eq}}$ for continental weathering, limiting the importance of kinetic controls on dissolution. In that case, unless the flow rate of water through hydrothermal systems is temperature dependent (analogous to the temperature dependence of runoff on continents), there might be limited scope for seafloor weathering to act as a thermostat. This idea could be explored with reactive transport modeling of seafloor hydrothermal systems. Regardless, the potential for alteration of oceanic crust to stabilize planetary climate is an important subject for further study, and it could have a strong impact on the effective outer edge of the HZ.

Another seafloor process left out of our habitability assessment is "reverse weathering," a process by which authigenic clay formation on the seafloor absorbs some fraction of the cations delivered to the ocean by silicate weathering, preventing the absorbed cations from forming carbonates to sequester $\mathrm{CO}_{2}$ from the atmosphere-ocean system (e.g., Mackenzie \& Garrels 1966; Dunlea et al. 2017; Isson \& Planavsky 2018; Trower \& Fischer 2019). At greater global rates of reverse weathering, fewer moles of $\mathrm{CO}_{2}$ are 
sequestered per unit silicate weathering, forcing a planet to warm up compared to the no-reverse-weathering case so that enough cations are delivered to the ocean to form carbonates and balance a given amount of outgassing. Isson \& Planavsky (2018) recently proposed that reverse weathering may provide a stabilizing climate feedback, operating as a complement to the silicate weathering feedback throughout the Precambrian on Earth. If reverse weathering accelerates under high-pH (low-pCO $\mathrm{CO}_{2}$ ) conditions, then its warming effect should become more pronounced when $\mathrm{CO}_{2}$ decreases and less pronounced when $\mathrm{CO}_{2}$ increases, increasing the stability of climate to changes in outgassing (and land fraction; Isson \& Planavsky 2018). However, the exact functional form of the $\mathrm{pH}$ dependence of reverse weathering reactions is not currently well constrained, and the significance of the process as a player in Earth's past climate may well have been moderate (Krissansen-Totton \& Catling 2020).

Another shortcoming of our study is the abstraction of inherently three-dimensional processes like precipitation and runoff on continents into zero-dimensional parameterizations. This was appropriate for a first attempt at understanding the implications of the MAC formulation for planetary weathering behavior, but examination of our conclusions with higherdimensional models would be worthwhile. Previous threedimensional GCM global weathering studies have used the WHAK formulation (e.g., Donnadieu et al. 2006; Edson et al. 2012; Paradise \& Menou 2017; Paradise et al. 2020). Expanding beyond our zero-dimensional models would allow us to quantify the importance of things like continental configuration (e.g., Lewis et al. 2018), atmospheric circulation (e.g., Komacek \& Abbot 2019), clouds (Komacek \& Abbot 2019), and rotation rate (e.g., Yang et al. 2014; Jansen et al. 2019) for planetary precipitation, runoff, and weathering behavior. Importantly, a full GCM study would naturally provide self-consistent treatments of the scalings of runoff and the energetic limit on precipitation with land fraction.

Related to the previous point, this study ignored the crucial and poorly constrained impacts of clouds on rocky planet climate. Clouds can have a profound impact on planetary albedo: water clouds on Earth reflect away approximately $47.5 \mathrm{~W} \mathrm{~m}^{-2}$ of insolation (Stephens et al. 2012), and simulations of tidally locked planets have found that thick cloud decks at the substellar point can reflect away enough starlight to double the instellation of the inner edge of the $\mathrm{HZ}$ for these planets (Yang et al. 2013). We implicitly included a cloud albedo effect by setting the surface albedo of our simulations to $a=0.3$, approximately equal to Earth's present-day albedo with clouds, but cloud albedo is intimately linked to atmospheric circulation, so a constant cloud albedo is a grave simplification. Clouds can also have a strong greenhouse effect: water clouds on Earth reduce OLR by approximately $26.4 \mathrm{~W} \mathrm{~m}^{-2}$ (Stephens et al. 2012). However, the particle size distribution for water clouds on exoplanets is completely unconstrained and has a strong impact on their net radiative effect (Komacek \& Abbot 2019). In addition to water clouds, $\mathrm{CO}_{2}$ ice clouds might be present in the atmospheres of planets with thick $\mathrm{CO}_{2}$ atmospheres (e.g., Forget \& Pierrehumbert 1997). $\mathrm{CO}_{2}$ ice clouds have been found to have strong impacts on albedo and OLR through their scattering properties in the visible and near-infrared (Forget \& Pierrehumbert 1997; Pierrehumbert \& Erlick 1998), although the magnitude of their effect may have been overestimated (Kitzmann 2016).

\subsection{Conclusions}

In this study, we applied the weathering framework developed by Maher \& Chamberlain (2014) and extended by Winnick \& Maher (2018) to evaluate rocky planet climate stability across a range of instellations and parameter choices. The MAC weathering framework includes a thermodynamic limit on weathering product concentration in runoff $\left(C_{\mathrm{eq}}\right)$ that previous formulations based on WHAK have not considered. $C_{\text {eq }}$ is controlled by $\mathrm{pCO}_{2}$ through the thermodynamics of coupled silicate dissolution and clay precipitation. We also included an energetic limit on global precipitation because instellation drives precipitation through the generation of evaporation, meaning the total latent heat flux from global precipitation should not exceed globally averaged instellation (though precipitation may locally exceed absorbed surface instellation slightly due to turbulent heat fluxes from atmosphere to surface, an effect we ignored; see, e.g., Pierrehumbert 2002; O’Gorman \& Schneider 2008).

We found that the MAC formulation leads to runoff- and $\mathrm{pCO}_{2}$-controlled weathering on rocky planets, which leads to interesting changes in planetary weathering behavior compared to simulations with the WHAK formulation that are governed by the kinetics of silicate dissolution. Simulations using the MAC formulation have climates that are more sensitive to $\mathrm{CO}_{2}$ outgassing rate and land fraction, but they are much less sensitive to the details of silicate dissolution kinetics like temperature and $\mathrm{pCO}_{2}$ dependence. These differences are due to the control of weathering rates in the MAC formulation by the thermodynamic balance between silicate dissolution and clay precipitation instead of the kinetics of silicate dissolution. In the WHAK formulation, increasing temperature increases both runoff and silicate dissolution rate, which increases the ability of WHAK planets to modulate their weathering flux with changes in temperature. In the MAC formulation, temperature only has an impact on weathering through changes in runoff (as well as a mild dependence of $C_{\mathrm{eq}}$ on temperature, which we excluded from our calculations), which leads to a weaker temperature dependence of weathering compared to the exponential dependence displayed by WHAK. This means that decreases in land fraction or increases in volcanic outgassing require larger compensatory temperature changes for planets governed by MAC weathering than for WHAK planets.

We also found that planets governed by MAC-style weathering have climates sensitive to the parameterization of hydrology and surface properties like soil age and soil porosity. Changes to these parameters that make global weathering more effective at delivering cations to the ocean at a given temperature and $\mathrm{pCO}_{2}$ lead to cooler equilibrium climates and move the effective outer edge of the HZ closer to the star. The apparent sensitivity of equilibrium rocky planet climate to these parameters suggests that "Earth-like" planets may be sensitive to the shifts in surface properties that are likely on a tectonically active surface, e.g., changes in uplift or outgassing rate. There may be a significant risk of catastrophic transitions to moist greenhouse or snowball states through stochastic changes to the parameters that control weathering.

Lastly, we showed that the energetic limit on precipitation set by planetary instellation can unintuitively lead to increases in planetary temperature with decreases in instellation. This is because planets that have reached their maximum precipitation at a given instellation lose the feedback on climate provided by the modulation of weathering flux in response to temperature 
through changes in planetary precipitation. Simulations beyond the instellation where the energetic limit kicks in experience a linear decrease in runoff with reduced instellation, which forces $\mathrm{pCO}_{2}$ to increase to higher levels so that $C_{\mathrm{eq}}$ can increase to levels that allow runoff to carry enough solute for weathering to balance outgassing. This larger increase in $\mathrm{pCO}_{2}$ with a decrease in instellation in the energetically limited regime leads to the increase in temperature with reduced instellation in some regions of parameter space, though at sufficiently high partial pressures the increase in $\mathrm{pCO}_{2}$ can also lead to cooling as the increased Rayleigh scattering of a thicker atmosphere begins to outweigh the increased greenhouse effect.

In summary, the inclusion of energetic and thermodynamic limits in our simulations of continental silicate weathering on rocky, ocean-bearing exoplanets leads to a diversity of stable climates throughout the $\mathrm{HZ}$, ranging from $>350 \mathrm{~K}$ to freezing, depending on a complex interplay of poorly understood factors. This diversity has important implications for the potential of future telescope missions to infer the operation of the silicate weathering feedback in the $\mathrm{HZ}$ with population statistics (see Section 5.1.4). The use of the MAC formulation allows for the explicit incorporation of variables like lithology, hydrology, and soil properties into models of terrestrial planet weathering, which allows for the investigation of a wealth of interesting questions about planetary habitability and the coupling of atmospheres and crusts. Future studies of rocky planet climate and weathering should consider applying the MAC framework instead of, or in addition to, the WHAK framework.

We thank Dorian Abbot, Edwin Kite, Tim Lichtenberg, Ramses Ramirez, and an anonymous reviewer for insightful comments and suggestions that improved various versions of this manuscript. R.J.G. thanks the participants at the Rocky Worlds Workshop in Cambridge and the Bornö School on Climate and Conditions for Life on Early Earth and Other Planets for thoughtful discussions on some of the issues covered in this study. R.J.G. acknowledges scholarship funding from the Clarendon Fund and Jesus College, Oxford. R.T.P. is supported by European Research Council Advanced grant EXOCONDENSE (\#740963).

\section{ORCID iDs}

\section{R. J. Graham (iD https://orcid.org/0000-0001-9289-4416}

\section{References}

Abbot, D. S. 2016, ApJ, 827, 117

Abbot, D. S., Cowan, N. B., \& Ciesla, F. J. 2012, ApJ, 756, 178

Abbot, D. S., Voigt, A., \& Koll, D. 2011, JGRD, 116, D18103

Alekseyev, V. A., Medvedeva, L. S., Prisyagina, N. I., Meshalkin, S. S., \& Balabin, A. I. 1997, GeCoA, 61, 1125

Avice, G., Marty, B., \& Burgess, R. 2017, NatCo, 8, 15455

Bahcall, J. N., Pinsonneault, M., \& Basu, S. 2001, ApJ, 555, 990

Bandstra, J. Z., \& Brantley, S. L. 2008, Kinetics of Water-Rock Interaction (Berlin: Springer), 211

Batalha, N. E., Kopparapu, R. K., Haqq-Misra, J., \& Kasting, J. F. 2016, E\&PSL, 455, 7

Bean, J. L., Abbot, D. S., \& Kempton, E. M.-R. 2017, ApJL, 841, L24

Berner, R. 1994, AmJS, 294, 56

Berner, R. A. 1992, GeCoA, 56, 3225

Blake, R. E., Chang, S. J., \& Lepland, A. 2010, Natur, 464, 1029

Boucher, D. F., \& Alves, G. E. 1963, Dimensionless Numbers: For Fluid Mechanics Heat Transfer, Mass Transfer and Chemical Reaction (New York: American Institute of Chemical Engineers)

Brady, P., \& Gislason, S. 1997, GeCoA, 61, 965
Brady, P. V. 1991, JGRB, 96, 18101

Brady, P. V., \& Carroll, S. A. 1994, GeCoA, 58, 1853

Brantley, S. L., Kubicki, J. D., \& White, A. F. 2008, Kinetics of Water-Rock Interaction (Cham: Springer)

Caldeira, K. 1995, AmJS, 295, 1077

Carretier, S., Goddéris, Y., Martinez, J., Reich, M., \& Martinod, P. 2018, ESuD, 6, 217

Carroll, S. A., \& Knauss, K. G. 2005, ChGeo, 217, 213

Catling, D. C., \& Zahnle, K. J. 2020, SciA, 6, eaax1420

Charnay, B., le Hir, G., Fluteau, F., Forget, F., \& Catling, D. C. 2017, E\&PSL, 474, 97

Checlair, J. H., Abbot, D. S., Webber, R. J., et al. 2019a, arXiv:1903.05211

Checlair, J. H., Salazar, A. M., Paradise, A., Menou, K., \& Abbot, D. S. 2019b, ApJL, 887, L3

Chen, Y., \& Brantley, S. L. 1998, ChGeo, 147, 233

Collins, D. B. G., Bras, R., \& Tucker, G. E. 2004, JGRF, 109, F03004

Coogan, L. A., \& Dosso, S. E. 2015, E\&PSL, 415, 38

Coogan, L. A., \& Gillis, K. M. 2013, GGG, 14, 1771

Cowan, N. B., \& Abbot, D. S. 2014, ApJ, 781, 27

Cronin, T. W. 2014, JAtS, 71, 2994

de Wit, M. J., \& Furnes, H. 2016, SciA, 2, e1500368

Donnadieu, Y., Goddéris, Y., Pierrehumbert, R., et al. 2006, GGG, 7

Dorn, C., Noack, L., \& Rozel, A. 2018, A\&A, 614, A18

Dunlea, A. G., Murray, R. W., Ramos, D. P. S., \& Higgins, J. A. 2017, NatCo, 8,844

Dunne, T. 1978, Natur, 274, 244

Edson, A. R., Kasting, J. F., Pollard, D., Lee, S., \& Bannon, P. R. 2012, AsBio, 12,562

Ferrier, K. L., \& Kirchner, J. W. 2008, E\&PSL, 272, 591

Flament, N., Coltice, N., \& Rey, P. F. 2008, E\&PSL, 275, 326

Foley, B. J. 2015, ApJ, 812, 36

Foley, B. J. 2019, ApJ, 875, 72

Foley, B. J., \& Smye, A. J. 2018, AsBio, 18, 873

Forget, F., \& Pierrehumbert, R. T. 1997, Sci, 278, 1273

France-Lanord, C., \& Derry, L. A. 1997, Natur, 390, 65

Gabet, E. J., \& Mudd, S. M. 2009, Geo, 37, 151

Gaillardet, J., Dupré, B., Louvat, P., \& Allegre, C. 1999, ChGeo, 159, 3

Gaudi, B. S., Seager, S., Mennesson, B., et al. 2020, The Habitable Exoplanet Observatory (HabEx) Mission Concept Study Final Report, https://www. jpl.nasa.gov/habex/pdf/HabEx-Final-Report-Public-Release-LINKED0924.pdf

Gerlach, T. 2011, EOSTr, 92, 201

Golubev, S. V., Pokrovsky, O. S., \& Schott, J. 2005, ChGeo, 217, 227

Haqq-Misra, J., Kopparapu, R. K., Batalha, N. E., Harman, C. E., \& Kasting, J. F. 2016, ApJ, 827, 120

Hart, M. H. 1979, Icar, 37, 351

Hayworth, B. P., Kopparapu, R. K., Haqq-Misra, J., et al. 2020, Icar, 113770

Heimsath, A. M., Chappell, J., Dietrich, W. E., Nishiizumi, K., \& Finkel, R. C. 2000, Geo, 28, 787

Held, I. M., \& Soden, B. J. 2006, JCli, 19, 5686

Henderson-Sellers, B. 1984, QJRMS, 110, 1186

Höning, D., Hansen-Goos, H., Airo, A., \& Spohn, T. 2014, P\&SS, 98, 5

Höning, D., \& Spohn, T. 2016, PEPI, 255, 27

Höning, D., Tosi, N., Hansen-Goos, H., \& Spohn, T. 2019, PEPI, 287, 37

Hren, M., Tice, M., \& Chamberlain, C. 2009, Natur, 462, 205

Huang, S.-S. 1959, AmSci, 47, 397

Ibarra, D. E., Caves, J. K., Moon, S., et al. 2016, GeCoA, 190, 265

Isson, T. T., \& Planavsky, N. J. 2018, Natur, 560, 471

Jagoutz, O., Macdonald, F. A., \& Royden, L. 2016, PNAS, 113, 4935

Jansen, T., Scharf, C., Way, M., \& del Genio, A. 2019, ApJ, 875, 79

Johnson, B. W., \& Wing, B. A. 2020, NatGe, 13, 243

Kadoya, S., \& Tajika, E. 2014, ApJ, 790, 107

Kadoya, S., \& Tajika, E. 2019, ApJ, 875, 7

Kadoya, S., Tajika, E., \& Watanabe, Y. 2014, in IAU Symp. 293, Formation, Detection, and Characterization of Extrasolar Habitable Planets (Cambridge: Cambridge Univ. Press), 319

Kasting, J. F. 1988, Icar, 74, 472

Kasting, J. F., \& Holm, N. G. 1992, E\&PSL, 109, 507

Kasting, J. F., Toon, O. B., \& Pollack, J. B. 1988, SciAm, 258, 90

Kasting, J. F., Whitmire, D. P., \& Reynolds, R. T. 1993, Icar, 101, 108

Kite, E., Gaidos, E., \& Manga, M. 2011, ApJ, 743, 41

Kite, E. S., \& Ford, E. B. 2018, ApJ, 864, 75

Kitzmann, D. 2016, ApJL, 817, L18

Knauss, K. G., Nguyen, S. N., \& Weed, H. C. 1993, GeCoA, 57, 285

Kodama, T., Genda, H., O'ishi, R., Abe-Ouchi, A., \& Abe, Y. 2019, JGRE, 124,2306 
Kodama, T., Nitta, A., Genda, H., et al. 2018, JGRE, 123, 559

Komacek, T. D., \& Abbot, D. S. 2016, ApJ, 832, 54

Komacek, T. D., \& Abbot, D. S. 2019, ApJ, 871, 245

Kopparapu, R. K. 2013, ApJL, 767, L8

Kopparapu, R. K., Ramirez, R., Kasting, J. F., et al. 2013, ApJ, 765, 131

Krissansen-Totton, J., Arney, G. N., \& Catling, D. C. 2018, PNAS, 115, 4105

Krissansen-Totton, J., \& Catling, D. C. 2017, NatCo, 8, 15423

Krissansen-Totton, J., \& Catling, D. C. 2020, E\&PSL, 537, 116181

Kump, L. R. 2018, RSPTA, 376, 20170078

Kump, L. R., \& Arthur, M. A. 1997, Tectonic Uplift and Climate Change (Berlin: Springer), 399

Kump, L. R., Brantley, S. L., \& Arthur, M. A. 2000, AREPS, 28, 611

Larsen, I. J., Almond, P. C., Eger, A., et al. 2014a, Sci, 343, 637

Larsen, I. J., Montgomery, D. R., \& Greenberg, H. M. 2014b, Geo, 42, 527

le Hir, G., Donnadieu, Y., Godderis, Y., et al. 2009, E\&PSL, 277, 453

Leconte, J., Forget, F., Charnay, B., Wordsworth, R., \& Pottier, A. 2013, Natur, 504, 268

Lenardic, A., Jellinek, A., Foley, B., O’Neill, C., \& Moore, W. 2016, JGRE, 121,1831

Lewis, N. T., Lambert, F. H., Boutle, I. A., et al. 2018, ApJ, 854, 171

Macdonald, F. A., Swanson-Hysell, N. L., Park, Y., Lisiecki, L., \& Jagoutz, O. 2019, Sci, 364, 181

Mackenzie, F. T., \& Garrels, R. M. 1966, AmJS, 264, 507

Maher, K., \& Chamberlain, C. 2014, Sci, 343, 1502

Maher, K., Steefel, C. I., DePaolo, D. J., \& Viani, B. E. 2006, GeCoA, 70, 337

Maher, K., Steefel, C. I., White, A. F., \& Stonestrom, D. A. 2009, GeCoA, 73,2804

Malmström, M. E., Destouni, G., Banwart, S. A., \& Strömberg, B. H. 2000, EnST, 34, 1375

Menou, K. 2015, E\&PSL, 429, 20

Montgomery, D. R., \& Brandon, M. T. 2002, E\&PSL, 201, 481

Neil, A. R., \& Rogers, L. A. 2020, ApJ, 891, 12

Noack, L., Godolt, M., von Paris, P., et al. 2014, P\&SS, 98, 14

Noack, L., Rivoldini, A., \& van Hoolst, T. 2017, PEPI, 269, 40

Oelkers, E. H., \& Schott, J. 2001, GeCoA, 65, 1219

O’Gorman, P. A., \& Schneider, T. 2008, JCli, 21, 3815

Ojakangas, R. W., Srinivasan, R., Hegde, V., Chandrakant, S., \& Srikantia, S. 2014, CSci, 106, 387, https://www.jstor.org/stable/24099899

Oki, T., Agata, Y., Kanae, S., et al. 2001, Hydrol. Sci. J., 46, 983

O’Neill, C. 2012, AuJES, 59, 189

Oxburgh, R., Drever, J. I., \& Sun, Y.-T. 1994, GeCoA, 58, 661

Palandri, J. L., \& Kharaka, Y. K. 2004, A Compilation of Rate Parameters of Water-mineral Interaction Kinetics for Application to Geochemical Modeling, Tech. Rep.,, Geological Survey Menlo Park CA

Paradise, A., \& Menou, K. 2017, ApJ, 848, 33

Paradise, A., Menou, K., Valencia, D., \& Lee, C. 2020, JGRE, 124, 2087

Pascucci, I., Mulders, G. D., \& Lopez, E. 2019, ApJL, 883, L15

Perron, J. T. 2017, AREPS, 45, 561

Peters, N. 1984, in U.S. Geological Survey Water Supply, Paper 2228 (Washington, DC: USGS), 39

Pierrehumbert, R., \& Erlick, C. 1998, JAtS, 55, 1897
Pierrehumbert, R., \& Gaidos, E. 2011, ApJL, 734, L13

Pierrehumbert, R. T. 2002, Natur, 419, 191

Pierrehumbert, R. T. 2010, Principles of Planetary Climate (Cambridge: Cambridge Univ. Press)

Pierrehumbert, R. T. 2011, PhT, 64, 33

Rafiei, M., \& Kennedy, M. 2019, NatCo, 10, 1

Ramirez, R. 2018, Geosc, 8, 280

Ramirez, R. M. 2017, Icar, 297, 71

Ramirez, R. M. 2020, MNRAS, 494, 259

Ramirez, R. M., \& Levi, A. 2018, MNRAS, 477, 4627

Raymo, M. E., \& Ruddiman, W. F. 1992, Natur, 359, 117

Rimstidt, J. D., Brantley, S. L., \& Olsen, A. A. 2012, GeCoA, 99, 159

Rosing, M. T., Bird, D. K., Sleep, N. H., \& Bjerrum, C. J. 2010, Natur, 464, 744

Rosing, M. T., Bird, D. K., Sleep, N. H., Glassley, W., \& Albarede, F. 2006, PPP, 232, 99

Rushby, A. J., Johnson, M., Mills, B. J., Watson, A. J., \& Claire, M. W. 2018 , AsBio, 18, 469

Sagan, C., \& Mullen, G. 1972, Sci, 177, 52

Schaefer, L., \& Sasselov, D. 2015, ApJ, 801, 40

Schott, J., \& Berner, R. A. 1985, The Chemistry of Weathering (Berlin: Springer), 35

Siever, R. 1968, Sedim, 11, 5

Stephens, G. L., Li, J., Wild, M., et al. 2012, NatGe, 5, 691

Stephens, G. L., O’Brien, D., Webster, P. J., et al. 2015, RvGeo, 53, 141

Sun, X., Higgins, J., \& Turchyn, A. V. 2016, MGeol, 373, 64

Tajika, E. 2007, EP\&S, 59, 293

The LUVOIR Team, L. 2019, arXiv:1912.06219

Tinetti, G., Meadows, V. S., Crisp, D., et al. 2006, AsBio, 6, 881

Trower, E. J., \& Fischer, W. W. 2019, SedG, 384, 1

Turbet, M. 2020, arXiv:2005.06512

Turbet, M., Forget, F., Leconte, J., Charnay, B., \& Tobie, G. 2017, E\&PSL, 11,11

Turyshev, S. G., Shao, M., Toth, V. T., et al. 2020, arXiv:2002.11871

Velbel, M. A. 1993, ChGeo, 105, 89

von Blanckenburg, F., Bouchez, J., Ibarra, D. E., \& Maher, K. 2015, NatGe, 8,538

Walker, J. C. G., Hays, P. B., \& Kasting, J. F. 1981, JGR, 86, 9776

Watanabe, Y., Tajika, E., \& Kadoya, S. 2014, in IAU Symp. 293, Formation, Detection, and Characterization of Extrasolar Habitable Planets (Cambridge: Cambridge Univ. Press), 333

Weissbart, E. J., \& Rimstidt, J. D. 2000, GeCoA, 64, 4007

Welch, S., \& Ullman, W. 1996, GeCoA, 60, 2939

West, A. J. 2012, Geo, 40, 811

West, A. J., Galy, A., \& Bickle, M. 2005, E\&PSL, 235, 211

White, A. F., \& Brantley, S. L. 2003, ChGeo, 202, 479

Winnick, M. J., \& Maher, K. 2018, E\&PSL, 485, 111

Xie, P. P., \& Arkin, P. A. 1997, BAMS, 78, 2539

Yang, H., Komacek, T. D., \& Abbot, D. S. 2019, ApJL, 876, L27

Yang, J., Boué, G., Fabrycky, D. C., \& Abbot, D. S. 2014, ApJL, 787, L2

Yang, J., Cowan, N. B., \& Abbot, D. S. 2013, ApJL, 771, L45

Yang, J., Peltier, W. R., \& Hu, Y. 2012, CliPa, 8, 907 